\title{
Insights into the dynamics of wake flame in a freely falling droplet
}

Cite as: Phys. Fluids 33, 123306 (2021); https://doi.org/10.1063/5.0073545

Submitted: 01 October 2021 • Accepted: 08 November 2021 • Published Online: 02 December 2021

Gautham Vadlamudi, S. K. Thirumalaikumaran and (Daptarshi Basu

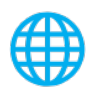

View Online
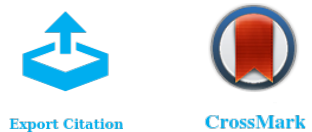

\section{ARTICLES YOU MAY BE INTERESTED IN}

Efficacy of homemade face masks against human coughs: Insights on penetration, atomization, and aerosolization of cough droplets

Physics of Fluids 33, 093309 (2021); https://doi.org/10.1063/5.0061007

Suppression of Leidenfrost effect on superhydrophobic surfaces

Physics of Fluids 33, 122104 (2021); https://doi.org/10.1063/5.0064040

Evaporation flow characteristics of airborne sputum droplets with solid fraction: Effects of humidity field evolutions

Physics of Fluids 33, 123308 (2021); https://doi.org/10.1063/5.0076572

\section{Physics of Fluids}




\title{
Insights into the dynamics of wake flame in a freely falling droplet
}

\author{
Cite as: Phys. Fluids 33, 123306 (2021); doi: 10.1063/5.0073545 \\ Submitted: 1 October 2021 - Accepted: 8 November 2021 . \\ Published Online: 2 December 2021
}

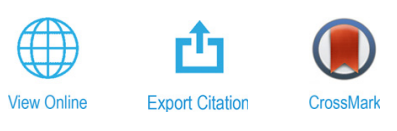

\author{
Gautham Vadlamudi, (D) S. K. Thirumalaikumaran,' and Saptarshi Basu',2,a) (D)
}

\section{AFFILIATIONS}

${ }^{7}$ Department of Mechanical Engineering, Indian Institute of Science, Bangalore 560012, India

${ }^{2}$ Interdisciplinary Centre for Energy Research (ICER), Indian Institute of Science, Bangalore 560012, India

${ }^{a)}$ Author to whom correspondence should be addressed: sbasu@iisc.ac.in

\begin{abstract}
The combustion of a freely falling dodecane droplet has been studied experimentally in a droptower-like facility under ambient conditions. A unique ignition mechanism is used by igniting the droplet in pendant mode and releasing it to fall freely. This unveils a different type of droplet wake flame behavior which is explored in this study. Initially, the droplet flame transitions from fully enveloped to a wake flame configuration due to forward extinction. The wake flame has similar characteristics as a laminar lifted triple-flame. As the droplet accelerates, the flame stand-off increases continuously. The change in wake flame topology and intensity occurs in two different regimes corresponding to different droplet diameters. A new non-dimensional parameter has been derived to account for the local balance between buoyancy and momentum diffusion that alters the fuel availability. To explain the flame topological evolutions and transitions for different droplet diameters and Reynolds numbers, a theoretical formulation has been proposed based on the momentum diffusion from surrounding due to relative motion. Further, at very high Reynolds number, flame stretching or shedding regime occurs, causing momentary spikes in flame intensity due to the interaction with asymmetric vortex shedding induced by the Bernard-Von Karman instability. Interestingly, the flame shedding height follows the buoyant flickering scaling, even for the momentum-dominant droplet wake flame. Additionally, the circulation build-up mechanisms are shown to be responsible for the flame shedding events for droplet wake flame at high Reynolds number.
\end{abstract}

Published under an exclusive license by AIP Publishing. https://doi.org/10.1063/5.0073545

\section{NOMENCLATURE}

$\begin{aligned} A_{d} & \text { Instantaneous line-of-sight droplet area } \\ A_{f} & \text { Instantaneous line-of-sight flame area } \\ D & \text { Flame width } \\ d & \text { Droplet diameter } \\ f & \text { Frequency }(\mathrm{Hz}) \\ g & \text { Acceleration due to gravity } \\ h & \text { Flame height } \\ I & \text { Flame intensity } \\ \dot{\boldsymbol{m}}_{a} & \text { Mass flow rate of oxidizer } \\ \dot{\boldsymbol{m}}_{f} & \text { Mass flow rate of fuel } \\ \dot{Q}_{a} & \text { Volume flow rate of oxidizer, air } \\ \dot{Q}_{f} & \text { Volume flow rate of fuel } \\ \dot{R e}_{e} & \text { Reynolds number } \\ S t & \text { Strouhal number } \\ T_{\text {air }} & \text { Ambient temperature } \\ T_{D D \text { vapor }} & \text { Dodecane vapor temperature } \\ \Delta T & \text { Temperature difference across the shear layer }\end{aligned}$

$v$ Droplet velocity

$\mathrm{v}_{a, \text { advection, } D} \quad$ Advection velocity of air across shear layer

$\mathrm{v}_{a, \text { momtDiff, }}$ Velocity scale associated with momentum diffusion

$\mathrm{v}_{\text {buoyancy,d }} \quad$ Natural convection velocity scale

$\mathrm{v}_{\text {core }}$ Core velocity of the vapor stream

$\mathbf{v}_{D D, \text { diffusion, } D}$ Diffusion velocity of dodecane vapor across shear layer

$\mathrm{v}_{f} \quad$ Flow velocity near the flame

$\mathrm{v}_{\text {flame }}$ Velocity of the flame with respect to ground

$\mathrm{v}_{N C} \quad$ Velocity scale of natural convection

\section{Symbols}

$\alpha$ Parameter that represents extent of momentum diffusion

$\beta$ Thermal expansion coefficient

$\Gamma$ Circulation

$\delta \quad$ Flame stand-off distance

$\rho_{a} \quad$ Density of oxidizer, air

$\rho_{f} \quad$ Density of fuel 


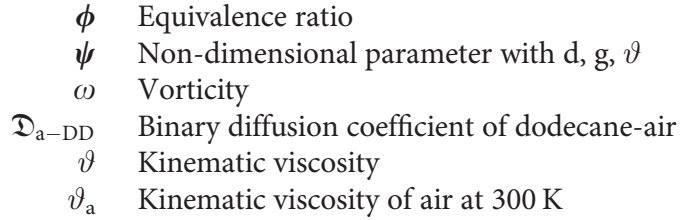

\section{Abbreviations}

BVK Bernard-Von Karman

Re Reynolds Number

Ri Richardson Number

\section{INTRODUCTION}

Spray combustion is one of the major modes of energy production across a multitude of applications ranging from power generation, basic transportation, to aero propulsion. The combustion of individual liquid droplet is the building block of the spray combustion phenomenon. A fundamental understanding of physics involved, like atomization, vaporization, flame dynamics, and flow interactions at the droplet level, will offer deeper insights into the heat and mass transfer phenomena involved at the subgrid level. These local phenomena govern global dynamics such as heat release rate, combustion efficiency, pollutant formation, and different types of instabilities. ${ }^{1}$ In spray combustion, the local extinction and blow out phenomenon affect combustion efficiency significantly. These local extinction mechanisms occur after enveloped flame transitions into wake configuration leading to extinction or blow out. The current study directly addresses such local extinction or blow-out behavior which will offer insights into the phenomenon especially when wake flame evolution can potentially occur in different regimes.

In the current study, the wake flame behavior in freely falling droplet for lower Reynolds number $(R e)$ has been investigated for different droplet diameters. The combustion of moving droplet in wake configuration has not been studied widely in the literature, especially when the wake flame stand-off distance is significantly high. A unique ignition and droplet release mechanism has been used in the experiments to get insight into the aforementioned case. The freely falling burning droplet generally undergoes different types of phenomena like flame extinction, lifted flame-like behavior, flame shedding, microexplosions, etc., that are discussed hereafter.

Kim et al. $^{2}$ have studied the liftoff flame behavior in a co-flow jet experiment by varying initial temperature. The consequent liftoff height depends on stabilizing the lifted flame edge by the balance mechanism of local flow velocity and flame propagation speed. The non-linear relation between liftoff height and jet velocity has an exponent (n) that increases with the initial temperature. This has been attributed to the differential diffusion that affects the fuel mass fraction with temperature change. The lifted flame has a tribrachial structure which exhibits a lean premixed flame branch, a rich premixed flame branch (both formed because of the stratification of the flow field), while the third branch is the diffusion flame envelope. ${ }^{3,4}$ The flame liftoff height is a function of flow rate and nozzle diameters. Furthermore, the tribrachial flame stabilization mechanism was found to be similar to that of stoichiometric premixed flame. In the case of an attached flame, the width decreases, and height increases with increase in the flow rate. However, with increased flow rate, the flame height decreases for a lifted flame, and the flame width increases due to hydrodynamic laminar jet-like expansion with associated increase in liftoff. ${ }^{5}$ The rate of increase in flame liftoff height in non-premixed lifted flames depends on the triple flame propagation speed. ${ }^{6}$ The speed and curvature of triple flame are shown to be a function of upstream mixture fraction gradient. In an experimental study conducted by Balasubramaniyan et al., the hydrodynamic instability and blowoff dynamics of a turbulent lean-premixed flame stabilized behind a cylindrical bluff body has been investigated. ${ }^{7}$ Different regimes of flame oscillation and stabilization have been observed depending on the equivalence ratios $(\phi)$.

Sapkal has shown that in a non-premixed lifted flame, the liftoff height increases with increase in dilution of the fuel stream or with increase in the velocity. The higher intensity of the edge flame helps the flame to stabilize at higher velocities. ${ }^{8}$ The characteristics of nearfield lifted laminar triple flames are experimentally and numerically investigated by Qin et al. ${ }^{9}$ They showed that the lifted flame behavior in the near-field exhibits a linear relationship with flow velocity, unlike the highly non-linear behavior of the lifted flames in the far-field. Kim et al. have experimentally investigated the extinction characteristics and flame dynamics of a premixed flame by imparting large variation in axial velocity, which offers insight into the unsteady behavior near the flammability limit. ${ }^{10}$ The lifted jet diffusion flame stabilizes when local velocity is close to premixed laminar flame speed and does not exceed 3SL (where $S_{L}$ is flame propagation speed). ${ }^{11}$ The upstream velocity is observed to decelerate near the flame as the streamlines diverge before accelerating behind the flame front. The leading-edge flame is reported to exhibit triple flame like behavior. The aforementioned conclusions about lifted flame from the literature were derived predominantly in context of a flame stabilized near the tip of a burner nozzle or in fully lifted configuration. Besides, these conclusions still hold good for a droplet flame exhibiting similar configuration or behavior.

Researchers have also investigated the effect of external forcing on the flame shape and heat release. Pandey et al. have shown that in the case of droplet flame, the flame heat release oscillation exhibits intrinsic convective length scales $(\mathrm{h})$ that correspond to the frequency of oscillation $(f)$ through the scaling $f \sim \sqrt{g / h}$ (where, $g$ is acceleration due to gravity), and the spectral response evolves temporally due to decreasing droplet size $(d){ }^{12}$ The shedding height was shown to be independent of the excitation frequency and depends only on the velocity amplitude of forcing. In the case of pool fires and jet flames, researchers showed that the shedding frequency associated with vortex roll-up is proportional to $\sqrt{g / l_{o}}$, where $g$ is the acceleration due to gravity, and $l_{o}$ is the characteristic length scale. ${ }^{13-15}$ The vortex roll-up mechanism occurs due to buoyancy-induced instability, and these vortices detach when the circulation build-up reaches a critical value causing flame flickering. Faster the circulation build-up rate, shorter is the vortex shedding height. The flame shedding and pinch-off events occur under external excitation because of circulation build-up.

In the case of pendant droplet studies, the filament affects the droplet combustion by acting as a heat sink, suppressing the evaporation rate and augmenting heat transfer from the flame to droplet. ${ }^{16}$ Hence, contactless droplet combustion studies like freely falling droplets, levitated droplets, etc., are essential to investigate the fundamental mechanisms governing spray combustion. Researchers have conducted many experimental studies on freely falling burning droplets. 
Guerrieri et al. have conducted drop-tower experiments for freely falling droplets in an oxygenated environment using metal nanoparticleladen hydrocarbon fuels to investigate the micro-explosion phenomenon and enhancement of heat release at various particle loading rates. ${ }^{17,18}$ Studies have also been conducted to investigate microexplosions in freely falling droplets by adding gas-generated Nitrocellulose particles to the fuel. Makino et al. have studied the ignition and combustion characteristics of a freely falling sodium droplet of different diameters and velocities. ${ }^{19}$ Wang et al. have investigated the combustion of freely falling multicomponent droplets to study the micro-explosion phenomenon occurring due to the boiling of volatile component. This increases the overall vaporization rate and heat release. ${ }^{20}$ The experiments have been performed by combusting methanol and alkane droplets after collision. ${ }^{21}$ The air entrapment during the collision provides heterogeneous nucleation sites causing early micro-explosion leading to lower droplet lifetime. Law et al. showed that there is a strong influence of convection (forced/natural/mixed) on the alkane burning rates. ${ }^{22}$ Experimental investigation has been performed to study the characteristics of expansion, ejection, and micro-explosion through a tubular furnace under different oxygen concentrations and temperatures for the bio-aviation fuel droplets. ${ }^{23}$ Further, a new model for micro-explosion under different oxygen concentrations is calculated and analyzed. The micro-explosions, internal ebullitions, and bulk droplet oscillations during the combustion of nanoparticle laden dodecane droplets have been investigated in pendant mode. ${ }^{24}$ The heterogeneous nucleation, bubble growth, and expulsion mechanisms were investigated in detail. Experiments have also been performed in contactless levitated mode to investigate the secondary atomization criteria of the nanoparticle laden fuel droplets using timescale analysis. ${ }^{25}$ Rao et al. have carried out experimental investigation to study the bubble dynamics and ligament-mediated atomization mechanisms during the combustion of multi-component droplets with significant volatility differential. ${ }^{26}$ All of the microexplosion, breakup phenomenon are essential to determine the droplet vaporization rate that further affects the heat release rate.

$\mathrm{Wu}$ et al. showed the burning rate is smaller in the case of wake flame. Lower ambient temperature delays the wake to envelope flame transition along with smaller burning rates. ${ }^{27}$ Huang et al. did a numerical study on contactless droplet flame under different gravitational fields. They showed that flame height, mass burning rate, Grashoff number, and Damkohler number increase with increase in droplet diameter. ${ }^{28} \mathrm{Wu}$ et al. have numerically derived the extinction criteria in convective environment for burning fuel particles. ${ }^{29}$ The extinction velocity increases with extinction diameter. In a convective environment, the enhancement of fuel evaporation rate and transport occurs. However, it has to overcome the heat transport away from the flame to avoid extinction. Tyurenkova et al. investigated the combustion characteristics of single droplet combustion under microgravity conditions. The cool flame occurrence after the visible flame extinction has been investigated. The presence of two flame surfaces corresponding to two reactions is proposed where both the flame surfaces exist at high temperatures. However, the first reaction ceases to occur in the low temperature regime, retaining only second flame surface which is the invisible cool flame responsible for the droplet regression post extinction of visible flame. ${ }^{30}$ A combustion model including chain reaction stages has been analytically investigated. Special transformation for Schvab Zeldovich model has been employed for the analysis along with the non-equilibrium model for the final stages of droplet regression. $^{31}$ The droplet regression models obtained in these studies are only applicable in the case of fully enveloped flame. However, as these reaction models alter the local flame propagation speed, they can be applied for the analysis of flame stabilization criteria. The nonequilibrium model is found to be effective for final stages of droplet regression at radius tending to zero in the case of fully enveloped droplet flame. It is not applicable for droplet wake flame.

The burning of spherical droplet in a hot oxidant convective flow is studied numerically using thin flame theory by Fendell et al. ${ }^{32}$ The flame position and surface mass transfer were shown to be a function of radial position and polar angle. The numerical simulations of flow over a sphere by Kurose et al. showed that the out flow from a sphere would reduce the total drag by reducing friction drag and increasing pressure drag. ${ }^{33}$ However, the momentum transport at the surface also contributes to the drag as the tangential momentum is convected by the radial velocity. ${ }^{34}$ The circulation inside the droplet decreases in the presence of outward normal velocity. For the freely falling droplet, drag initially increases with velocity, but later it decreases with diameter regression, and the maximum velocity is reached when drag balances the weight. ${ }^{35}$ As the droplet falls, the burning rate is enhanced by the convection of the evaporated fuel. The vorticity caused by the shear stress is convected downstream, which results in the reduction of drag. Jog et al. incorporated second order equations, which predict increased interfacial heat and mass transport, lower flame standoff, lower droplet lifetime, and smaller total drag, leading to asymmetrical vortical flow inside the droplet. ${ }^{36}$ The combustion experiments have been conducted by Balakrishnan et al. to investigate the enveloped flame shape characteristics in a mixed convective environment. ${ }^{37}$ The experiments have been done with flow velocity in the same direction as buoyancy in the first case and in the opposite direction for the other case. Theoretical predictions for burning rate and flame shape were advocated. All the studies as mentioned earlier predominantly focus on the lower droplet velocities corresponding to enveloped flame. Experiments were performed on small 400 and $70 \mu \mathrm{m}$ contactless fuel droplets having spherical flame both under microgravity and freely falling configurations. ${ }^{38}$ Extinction was observed to occur when the evaporation constant begins to decrease during later stages due to the reduction in Damkohler number. Additionally, soot formation is observed to decrease with decrease in initial droplet diameter.

The drop-tower experiments have been conducted with freely falling burning droplets by Pandey et al. ${ }^{39}$ for $2.2 \mathrm{~mm}$ alumina nanoparticle laden dodecane droplets. The effect of nanoparticle concentration along with hydrodynamic effects on the flame shape, heat release, and flame stabilization mechanisms has been investigated. The round jet analogy has been used to estimate the flame shape for the expanding flow around the wake flame stabilized at the rear stagnation point. The presence of wake flame in rear stagnation point leads to increase in viscosity of the hot gases, resulting in viscous dissipation of vorticity and suppression of Bernard-Von Karman (BVK) instability.

In the current study, drop-tower experiments are performed to study flame dynamics. The main-focus is to explain the different regimes of flame behavior due to the influence of flow field around a freely falling burning droplet. It is to be noted that the ignition mechanism used here differs from the previous experimental investigations in literature wherein the droplet was ignited while it was in motion. Here, the droplet is ignited at rest and then released to fall freely under 
gravity. So, this study unveils a unique and completely different type of wake flame behavior exhibited in a freely falling combusting droplet due to the temporally increasing flow velocity. The initial stages of flame dynamics discussed in this study can also offer further insights into the low droplet velocity regime in the literature ${ }^{39}$ that could not be investigated due to experimental limitations. This study also tries to explore the extinction/blow-out phenomena and shedding in a moving burning droplet. The local transition between premixed and nonpremixed flame structure has also been discussed that will provide insight into the sooting tendency in spray combustion.

\section{EXPERIMENTAL METHODOLOGY}

\section{A. Experimental set-up}

The combustion of a freely falling, low vapor pressure fuel droplet has been experimentally investigated using a drop-tower set-up under ambient conditions. The dodecane droplet is suspended at the junction of two needles that are mounted on a pair of linear solenoid actuators (each with $\sim 5 \mathrm{~mm}$ travel). The needle tips touch each other when both the solenoids are not activated. A heater (controlled by a PID-controller) is mounted on a heavy-duty linear solenoid actuator with a longer $(\sim 40 \mathrm{~mm})$ travel distance. The heater tip is aligned with the junction of the needles with $\sim 2 \mathrm{~mm}$ clearance. The entire set-up is then mounted on top of a drop-tower, as shown in Fig. 1.

A Photron SA5 HSS high-speed camera (coupled with a combination of $100 \mathrm{~mm}$ Tokina macrolens and $36 \mathrm{~mm}$ extension tube) is used with a Veritas light source for the shadowgraphy measurements. Another high-speed camera, Phantom Miro (coupled with $100 \mathrm{~mm}$ Tokina macrolens) has been used for flame intensity measurements. An in-house Arduino circuit is used to trigger, time, and synchronize all the different components in the system. The pendant droplet is ignited using a heater coil which is mounted on a heavy-duty linear solenoid. The heater-coil which is initially in retracted position is released forward for $600 \mathrm{~ms}$ and retracted when the Arduino is triggered. Once the heater retracts fully, both the needles are simultaneously retracted, releasing the burning dodecane droplet to fall freely due to gravity. As the combusting droplet falls, both the cameras are triggered accordingly for droplet shadowgraphy (at $7000 \mathrm{fps}$,
$1024 \times 1024$ resolution) and flame intensity imaging (at $3800 \mathrm{fps}$, $1024 \times 1024$ resolution $10 \mathrm{~ms}$ exposure) as the droplet passes through the respective ROI (each of size $4 \times 4 \mathrm{~cm}^{2}$ ). About 12 experimental runs were performed for each of the different ROIs (region of interests) located between $10 \mathrm{~cm}$ and $30 \mathrm{~cm}$ of distance below the needles. Experiments were performed for different droplet diameters $\sim(0.8-2.5 \mathrm{~mm})$ with the uncertainty in droplet diameter measurement found to be $\pm 0.02 \mathrm{~mm}$ based on the resolution of data acquisition. The data acquired across the different ROIs are consolidated and analyzed qualitatively and quantitatively to draw comprehensive conclusions regarding the flame evolution. It is to be noted that, due to the spatial orientation of cameras and light source, the fuel vapor trail in the aft end of the falling droplet scatters the light, which is also captured in flame imaging. However, due to experimental limitations, to detect the presence of yellow tip, the colored flame images are captured using a DSLR camera for a pendant droplet with similar co-flow as in the drop-tower.

\section{B. Image processing and data analysis of droplet shape}

The acquired droplet grayscale images are converted to binary, using MATLAB. The instantaneous droplet area and shape descriptors are extracted from these binary images. The droplet diameter $(d)$ is evaluated by equating the instantaneous droplet area to that of a circle, i.e., $A_{d}=\pi d^{2} / 4$.

\section{Flame imaging}

High-speed flame imaging enables quantitative measurement of the flame location, shape, and intensity that qualitatively indicates the flame heat release. By using the intensity thresholding technique, the flame intensity is isolated from the background. The flame boundary is isolated based on a cutoff intensity $(I)$. The pixels $(i$, ) having intensity $\geq I$ are assigned a binary value of 1 , and those with intensity $<I$ are allocated 0 . The resultant binary area comprising of pixels having value 1 is used to calculate the instantaneous line-of-sight flame area $\left(A_{f}\right)$. The intensities of all the pixels in the actual raw image
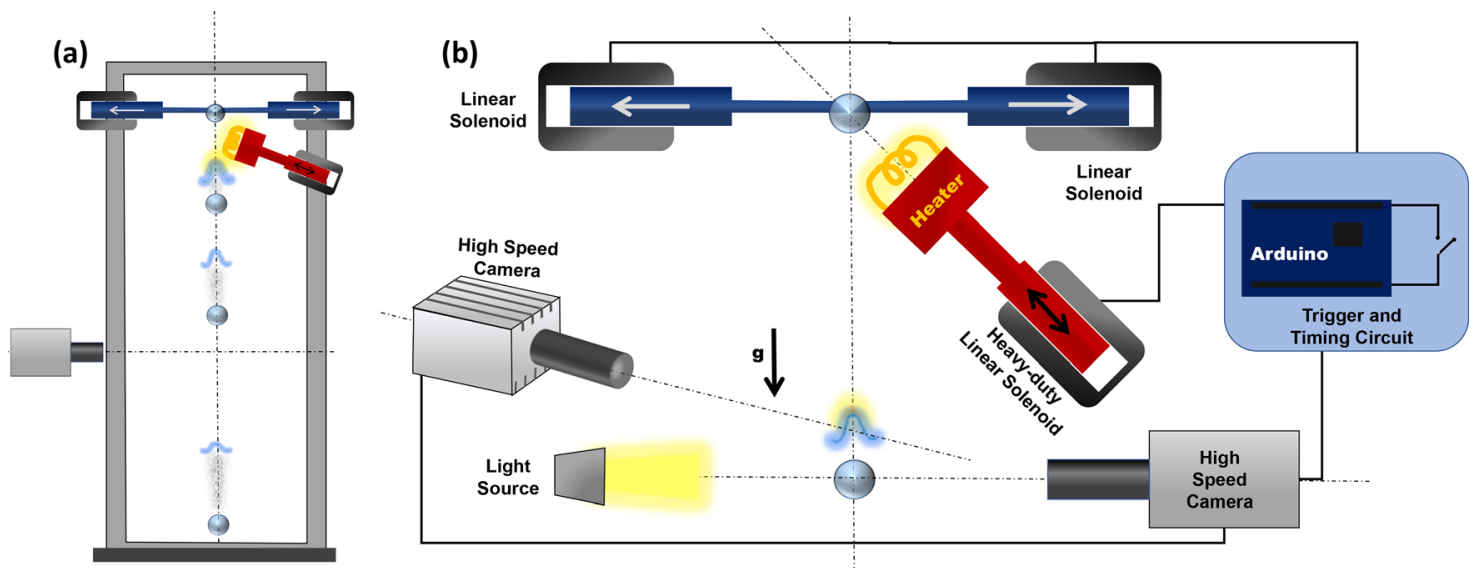

FIG. 1. The experimental setup. (a) Drop tower-like facility, with droplet ignition-release mechanism at the top and high-speed cameras mounted at specific height below the release point. (b) The enlarged depiction of the linear solenoid-actuated ignition mechanism and droplet release arrangement using retraction mechanism. Two high speed cameras were used for shadowgraphy (with backlighting) and flame imaging. All of the components are synchronized using timing and trigger circuit. 
corresponding to value 1 in this line-of-sight flame image are used to evaluate the flame intensity or luminosity for each frame.

\section{GLOBAL OBSERVATIONS}

As the droplet accelerates, the relative velocity of the surrounding gas phase continuously increases with time. Hence, the temporal variation of flame dynamics can be cast in terms of Reynolds number $(R e=d / \vartheta)$. In the current drop-tower experiments, after the droplet gets ignited using a heater coil and released, it accelerates under gravity from rest (see Fig. 1). Usage of this unique ignition mechanism to generate the freely falling burning droplet enables visualization of different new types of flame behavior. It also covers the closed tip wake flame behavior, especially at low $\operatorname{Re}<20$, which has not been investigated in the literature. $^{39}$ In current experiments, the unique ignition mechanism enables the sustenance of this closed tip wake flame even at higher Reynolds number which facilitates exploration of detailed physical insights. Figure 2 (Multimedia view) shows the experimental images of the freely falling burning droplet at different droplet diameters (d), where the flame is stabilized at the end of the vapor trail in the droplet wake.

With increasing Reynolds number, the droplet flame is observed to exhibit various topological evolutions (Fig. 2). Initially, after the droplet is released, transition occurs from a fully enveloped flame with a characteristic teardrop shape to a wake flame configuration beyond $R e>5$ due to local extinction at the forward stagnation point. The flame strain rate exceeds the critical strain rate at the forward stagnation point as the droplet velocity reaches $\sim 0.5 \mathrm{~m} / \mathrm{s}$. As the droplet accelerates further, natural convection becomes increasingly less

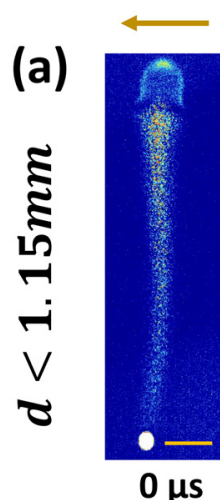

Diffusion flame

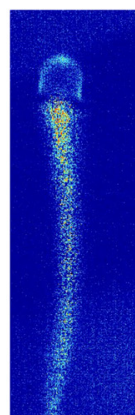

$5.2 \mu s$

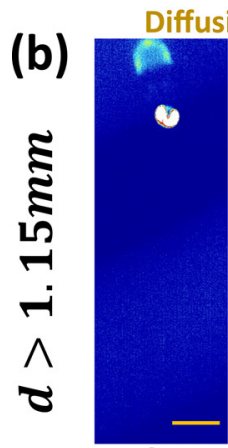

$0 \mu s$

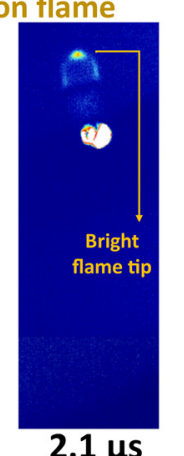

$2.1 \mu s$
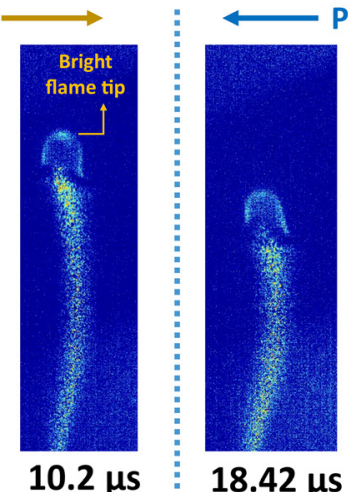

$18.42 \mu \mathrm{s}$
Premixed flame

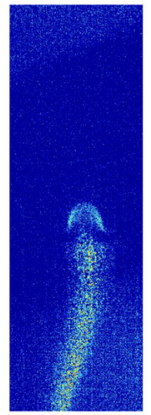

$23.8 \mu \mathrm{s}$

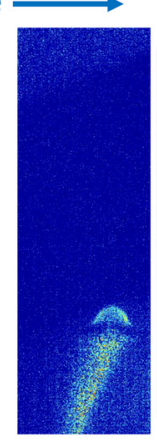

$34.2 \mu s$

Diffusion flame

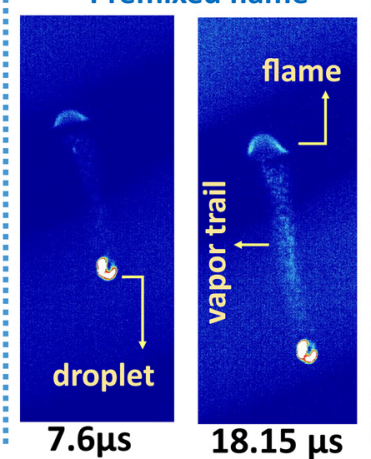

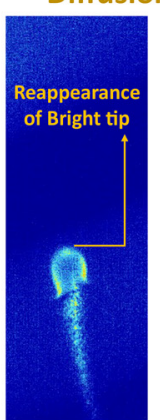

$34.2 \mu s$

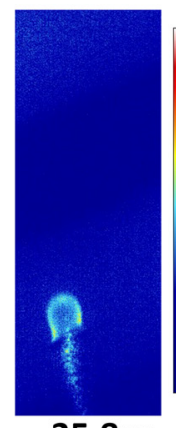

$35.8 \mu s$
$2000 C$

$1000 C$

2000

20000

2000

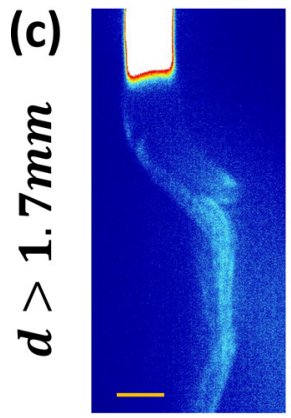

$31.32 \mu \mathrm{s}$

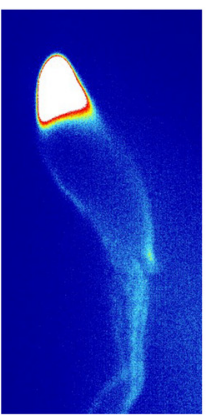

$36.57 \mu s$

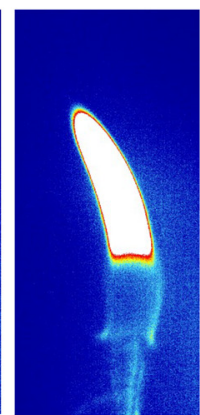

$41.84 \mu s$

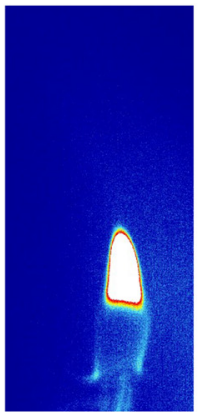

$44.42 \mu s$

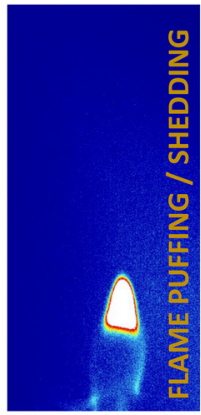

$46.84 \mu \mathrm{s}$
FIG. 2. Time series of flame imaging for different droplet diameters (d) of a freely falling burning droplet (with wake flame): (a) $d<1.15 \mathrm{~mm}$, (b) $d>1.15 \mathrm{~mm}$ with non-oscillating wake flame, and (c)d $>1.7 \mathrm{~mm}$ with oscillating wake flame. All the scale bars represent $2.5 \mathrm{~mm}$. The color bar represents the flame intensity for the respective regimes. Note that the luminosity range is selected specifically for the purpose of showing both the low intensity vapor trail and the flame shape with high intensity, leading to saturation at the flame tip (just for depiction purpose). Multimedia views: https://doi.org/10.1063/5.0073545.1; https://doi.org/10.1063/5.0073545.2; https:/ doi.org/10.1063/5.0073545.3 
dominant. This causes reduction in the size of the sooty yellow nonpremixed flame tip, in the range $5<R e<10 .{ }^{39}$ Consequently, as the Reynolds number increases further, the wake flame acts similar to a laminar lifted flame with co-flow, whose stand-off distance or liftoff height $(\delta)$ increases with flow velocity, as shown in Fig. 2. From the literature, it is known that the lifted flame exhibits a tribrachial structure at the leading-edge.

The wake flame observed is conjectured to have a tribrachial/triple flame like structure similar to the lifted flame, as shown in Fig. 2. In a triple-flame, there is a premixed flame front that tries to propagate upstream. $^{40}$ This premixed flame front is leaner on the outer edge on oxidizer side and is richer on the inner core, i.e., the fuel side. The equivalence ratio $(\phi)$ is observed to be varying continuously along the contour of this premixed flame wing due to stratification of fuel concentration. At the fuel-rich premixed flame branch (on the inside), the excess fuel diffuses further downstream and reacts with the oxidizer at the $\phi=1$ plane, forming a diffusion flame envelope. This diffusion flame anchors on the $\phi=1$ plane on the premixed flame wing. ${ }^{3}$ It is to be noted that if the inner premixed branch is fuel-rich, then the flame exhibits the characteristic yellow luminous diffusion tip, as shown in [Figs. 2(a) and 2(b)] (marked as diffusion flame). The excess fuel near the inner branch diffuses downstream and mixes with the oxidizer to form the diffusion flame envelope, which exhibits a characteristic yellow color due to higher soot formation tendency. The flame tip is non-premixed (diffusion) in nature due to fuel-rich zone at the core. However, when the inlet fuel mixture fraction becomes lean $(\boldsymbol{\phi}<\mathbf{1})$, the yellow diffusion tip disappears, as shown in Figs. 2(a) and 2(b) (marked as premixed flame). This process is hereafter referred to as the flame transition to fully premixed mode. The higher concentration gradient across the premixed flame wing will result in formation of a larger diffusion flame envelope. Hence, better mixing of the fuel and oxidizer upstream of the flame can also reduce the size of the yellow sooty flame tip. Similarly, an increase in fuel flow rate will increase the diffusion flame portion. ${ }^{41}$

Interestingly, when the droplet is suddenly released, the flame is not able to follow the droplet closely, causing the wake flame stand-off distance $(\delta)$ to increase continuously (see Fig. 2). For $R e>5$, increase in $\delta(\gg \mathrm{d})$ leads to decay of heat input rate to the droplet due to increase in distance from the flame. ${ }^{27}$ Hence, the fuel concentration necessary to sustain higher temperatures for the flame to be stabilized near the rear stagnation zone is no longer maintained. This causes further increase in stand-off distance as the droplet accelerates. Furthermore, the smaller flame is not able to maintain proximity to the droplet due to the lower fuel consumption rate. The small flame size is attributed to the lower fuel concentration available for the flame as a consequence of lower vaporization rate. The lower vaporization rate can be attributed to the low vapor pressure of the fuel or smaller droplet diameter.

The vapor field of fuel visible in the experimental images suggests that the wake flame is stabilized at the end of the fuel vapor trail (present aft side of the droplet) and follows the trail as the droplet falls (Fig. 2). The length of this vapor trail is observed to increase temporally reflecting the increasing trend of $\delta$. This behavior is mainly due to the continuous increase in relative velocity of the surrounding air, which pushes the flame away from the droplet, similar to that of a laminar lifted flame with a co-flow. ${ }^{2}$ It is observed that the droplet velocity ( $\sim 1 \mathrm{~m} / \mathrm{s}$ ) is faster than the falling velocity or spatial displacement velocity of the flame $(\sim 0.3-0.5 \mathrm{~m} / \mathrm{s})$. Therefore, it can be inferred that with respect to the droplet reference frame, the flame exhibits a receding relative velocity. Moreover, since the flame also has a downward velocity with respect to the ground, there is an upward motion of the surrounding air with respect to the flame.

\section{A. Effect of droplet diameter and necessity of new scaling parameter $(\psi)$}

The flame behavior is observed to be different for different ranges of diameters i.e., $d<1.15 \mathrm{~mm}, d>1.15 \mathrm{~mm}$, and $d>1.7 \mathrm{~mm}$ as shown in Fig. 2. Interestingly, for same droplet velocity or at the same time instant after release of the droplet, the flame shape and dynamics are observed to be different for various droplet diameters, as shown in Figs. 2 and 3(a). Even though the flame behavior is evolving with Reynolds number temporally, the droplet diameter affects the course of evolution of the flame dynamics locally. We hypothesize that the droplet diameter affects the direction of flame evolution by altering the local fuel availability through a delicate balance between buoyancy and the entrainment effects or momentum diffusion, as shown in Fig. 3(b). This local balance, coupled with the momentum effects of the flow $(R e)$, will dictate the dynamics of the droplet wake flame. This variation necessitates a new parameter that dictates the relative dominance of the buoyancy and momentum diffusion. Hence, a new nondimensional parameter is formulated, which is a function of droplet diameter $(\mathrm{d})$, kinematic viscosity of air $\left(\vartheta_{\mathrm{a}}\right)$, and acceleration due to gravity (g), as shown in Eqs. (1)-(4). Using dimensional analysis, $\psi$ is found to be the ratio of velocity scales associated with buoyancy and momentum diffusion, which is observed to increase with increase in droplet diameter (d),

$$
\begin{gathered}
\frac{d^{3 / 2} \sqrt{g}}{\vartheta_{a}} \sim \frac{d \sqrt{g d}}{\vartheta_{a}}, \\
f(\beta, \Delta T) \sim \sqrt{\beta \Delta T}, \\
{\left[\frac{d \sqrt{g d}}{\vartheta_{a}} * f(\beta, \Delta T)\right] \sim \psi,} \\
\psi \sim \frac{\sqrt{g \beta \Delta T d}}{\frac{\vartheta_{a}}{d}} \sim \frac{\mathbf{v}_{\text {buoyancy, } \boldsymbol{d}}}{\mathbf{v}_{\boldsymbol{a}, \text { momt } \text { Diff }, \boldsymbol{d}}}
\end{gathered}
$$$$
\text { (for flow near the droplet, } \quad \beta \sim \frac{1}{400} \mathrm{~K}, \quad \Delta T \sim\left[T_{D D \text { vapor }}-T_{\text {air }}\right] \mathrm{K} \text {, }
$$$$
\left.T_{\text {air }} \sim 300 \mathrm{~K} ; T_{\text {Dodecanevapor }} \sim 500 \mathrm{~K}\right) \text {. }
$$

We subsequently classify the flame behavior into different regimes, as depicted in Fig. 3(a). Since the direction of evolution of the flame dynamics is determined by non-dimensional parameter $(\psi)$ and the temporal evolution is dictated by Reynolds number, a regime map has been conceived. The Reynolds number (Re) of surrounding air with respect to the droplet diameter (d) is plotted on the $\mathrm{x}$-axis and the non-dimensional parameter $\psi$ on y-axis in the regime map, as shown in Fig. 3(a). The higher value of $\psi$ indicates the relative increase in importance of buoyancy effects. Hence, it can be inferred that for higher values of $\psi$, the tendency to form buoyant flame tip increases. This is also reflected in the experimental observations as flame 
(a)

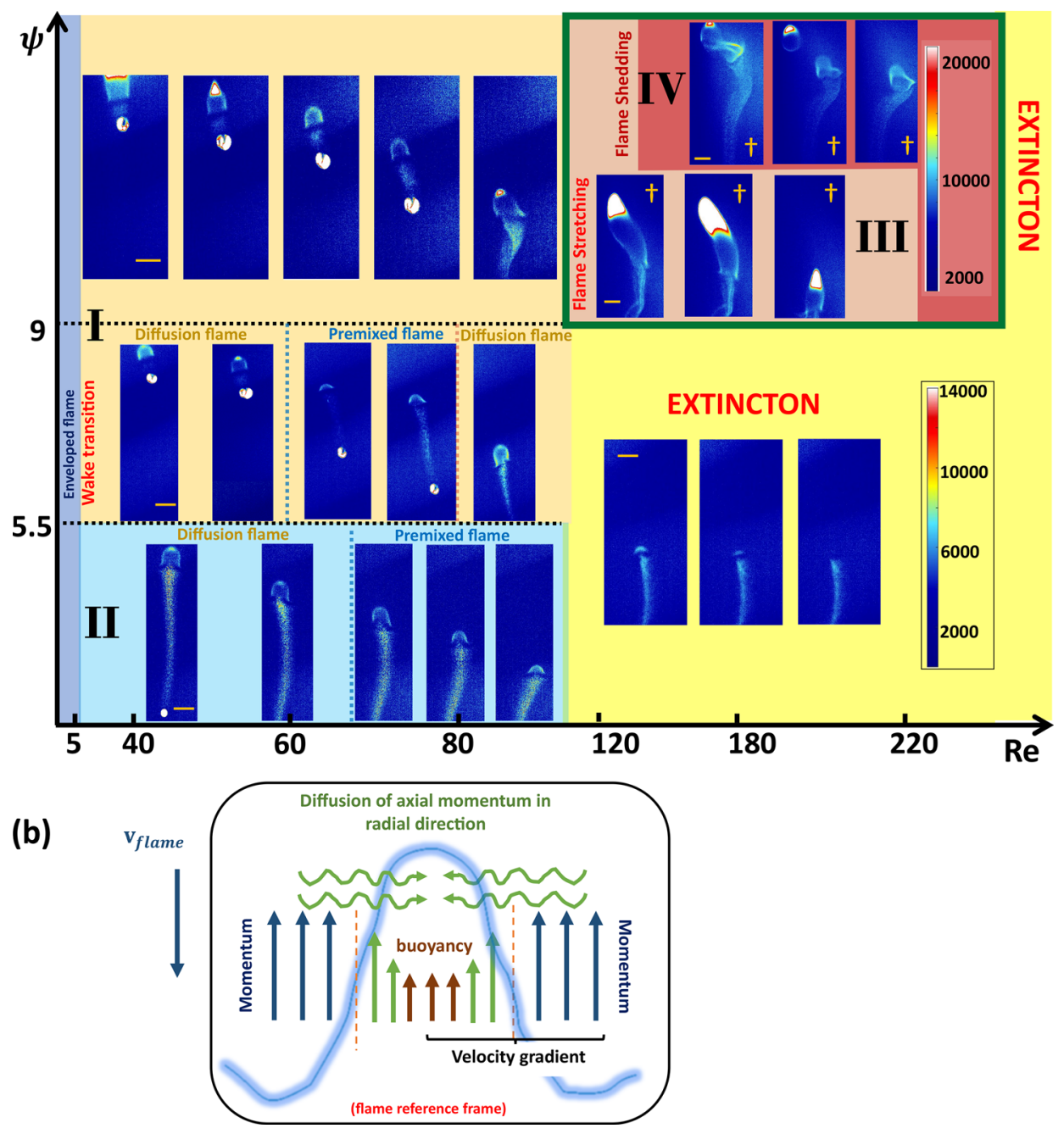

FIG. 3. (a) The experimental flame configuration regime map, non-dimensional parameter $(\psi)$ vs Reynolds number $(\boldsymbol{R e})$. The flame images are depicted in different regimes of the flame behavior. All the scale bars represent $2.5 \mathrm{~mm}$. The color bar shown at the top represents the flame intensity for the stretching/shedding regimes III and IV (enclosed by solid green rectangle and denoted by $\dagger$ ), and the color bar at the bottom represents the flame intensity for all the other regimes (I, II, and extinction) depicted in the plot. The wake flame topological behavior corresponding to $\psi>9$ (d $>1.7 \mathrm{~mm})$ for low Reynolds number is shown in Multimedia view. (b) The schematic showing the local balance between buoyancy and momentum diffusion. $v_{\text {flame }}$ represents the falling velocity of flame with respect to ground and all the velocities inside the enclosure are in the reference frame of the flame. Multimedia view: https://doi.org/10.1063/ 5.0073545 .4

transitions into yellow tip (diffusion mode) for higher values of $\psi$ even at higher velocity scales, as shown in Fig. 3(a).

\section{B. Effect of Reynolds number (Re) on flame dynamics in different regimes}

In $R e<60$ range, for all the droplet diameters, the wake flame exhibits the characteristic luminous yellow tip. The flame intensity is observed to decrease with increase in Reynolds number. Furthermore, at lower droplet diameter $(\mathrm{d}<1.15 \mathrm{~mm})$, i.e., $\psi<5.5$, the flame intensity decreases further with increase in Reynolds number. The characteristic yellow diffusion flame changes gradually into a pale blue premixed counterpart at $R e \sim 72$. For $R e>72$, the flame width $(D)$ increases and height $(h)$ decreases similar to the laminar jet flow behavior [see Fig. 2(a)]. This causes the vapor stream to become increasingly fuel lean due to air entrainment in the downstream direction. This flame evolution has been depicted in regime II of Fig. 3(a).
Finally, when sufficient fuel is no longer available at $\operatorname{Re} \sim(90$ to 100$)$, the flame starts to decay, leading to extinction.

On the other hand, for $d>1.15 \mathrm{~mm}$, i.e., $\psi>5.5$, after an initial decrease for $R e<60$, the flame intensity starts to increase beyond $R e>60$. The characteristic yellow diffusion tip diminishes and the flame changes gradually into fully premixed pale blue color at $R e \sim(60-67)$ due to air entrainment. However, the flame intensity is observed to increase with further increase in Re, leading to the reappearance of the characteristic yellow diffusion tip at $R e \sim 77$ [see Fig. 2(b)]. This transition to yellow diffusion tip at $R e \sim 77$ in the regime $\psi>5.5$ can also be supported by the higher value of $\psi$ when compared to $\psi>\mathbf{5 . 5}$ regime, suggesting increase in buoyancy effects. These flame transitions are shown in regime I of Fig. 3(a). Finally, beyond $R e>(110-120)$, the flame intensity starts to decrease as the flame extinction occurs. The temporal variation of the flame luminosity for both $\boldsymbol{\psi}>\mathbf{5 . 5}$ and $\boldsymbol{\psi}<\mathbf{5 . 5}$ regimes is shown in Figs. 4(a) and 4(b), respectively. Additionally, in both regimes I and II, the flame height exhibited small jitter-like high frequency oscillations at the 

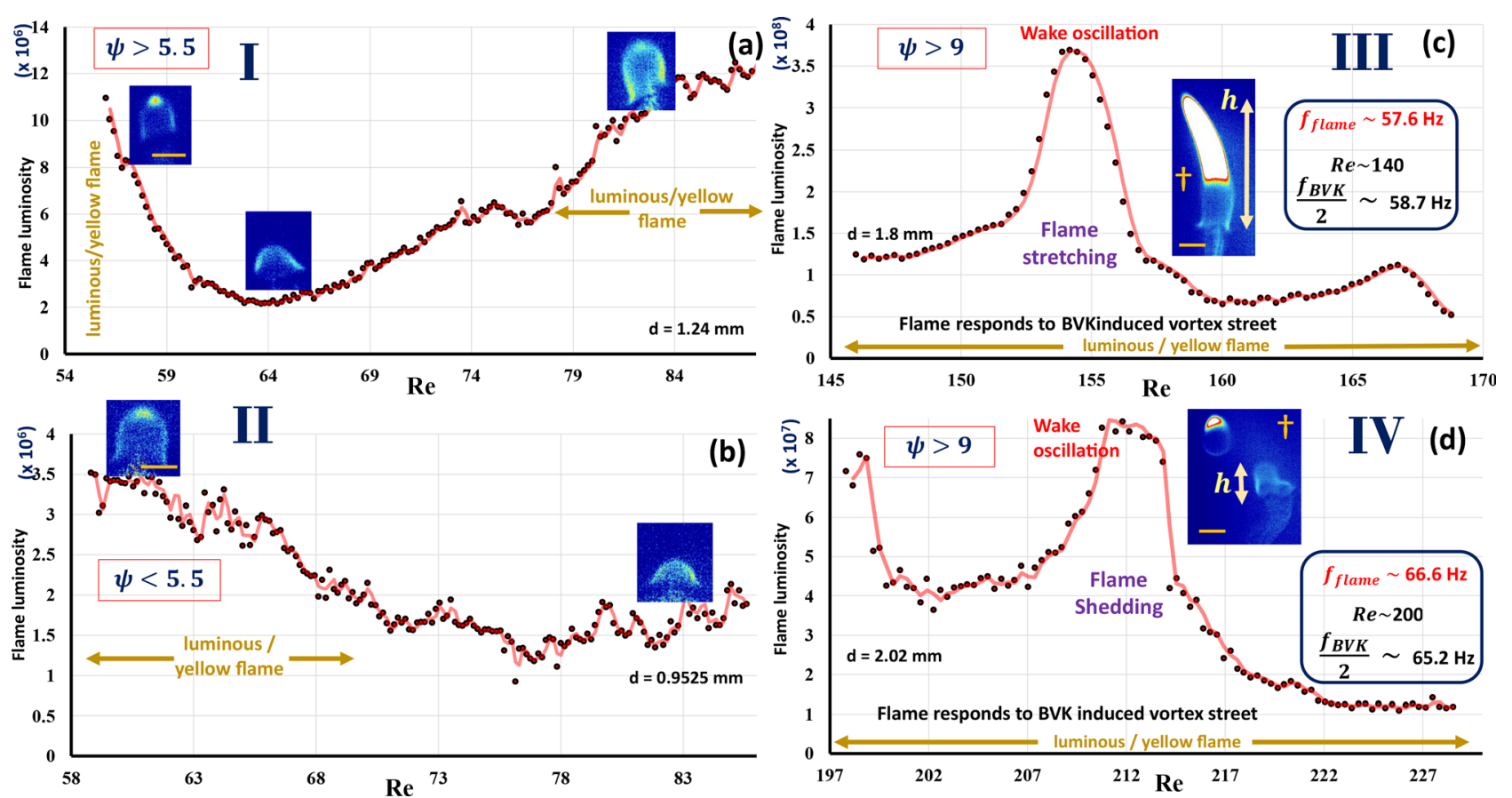

FIG. 4. The flame luminosity is plotted against Reynolds number (Re) for different regimes exhibiting different flame behaviors. (a) $\psi>\mathbf{5 . 5}$, (b) $\psi<\mathbf{5 . 5}$, (c) $\psi>\mathbf{9 - f l a m e}$ stretching, and (d) $\psi>$ 9-flame bulk shedding. All the scale bars represent $2.5 \mathrm{~mm}$. In subfigures (c) and (d), the flame shedding/stretching images are embedded and are denoted by the symbol $\dagger$. The flame shedding height $(h)$ for each case is indicated using double-arrow for regimes III and IV. The details of flame response frequency $\left(f_{\text {flame }}\right)$ due to interaction with Bernard-Von Karman (BVK) induced vortices and the calculated value of BVK vortex shedding frequency $\left(f_{B V K}\right)$ based on Reynolds number for corresponding runs have been shown in box for regimes III and IV.

flame tip reflected in the time series data [see Figs. 4(a) and 4(b)]. The schematic depicting the flame transitions and topological evolutions for regimes I and II is shown in Fig. 5.

However, for higher diameters $(d>1.7 \mathrm{~mm})$, i.e., $\boldsymbol{\psi}>\mathbf{9}$, the flame intensity follows a similar trend as $\psi>\mathbf{5 . 5}$ but the flame is sustained at even higher on Reynolds number due to higher fuel availability, and the extinction phase is shifted to higher $R e$ $>$ (200-240). Additionally, flame stretching/puffing phenomena are observed for $R e \sim(140$ to 170$)$ and flame bulk shedding at around $R e \sim 200$ followed by extinction, as shown in Figs. 2(c) and 3(a) regimes III and IV. The droplet wake flow field becomes asymmetric after a critical timescale with increasing velocities. ${ }^{42}$ The flame pinch-off events [Fig. 2(c)] are a consequence of the interaction of flame with Bernard-Von Karman (BVK) instabilityinduced asymmetric vortex shedding from the droplet. The vortexshedding disturbance has to travel all the way from the droplet toward the flame. The peaks present in Figs. 4(c) and 4(d) represent the flame pinch-off and bulk shedding events, respectively, for $\psi>9$. The flame in this regime is observed to possess a significantly longer $(\sim 10 d)$ luminous sooty yellow portion of the flame, suggesting that the hot gases accelerate over longer distances along the flame making the buoyancy effects important in addition to higher fuel availability. This is also indicated by the large $\psi$ value in these regimes III and IV. For all diameter ranges, when the flame ultimately enters the extinction phase, both the flame height $(h)$ and width $(D)$ decrease, as shown in Fig. 5 (Multimedia view).
Note that, in all the cases, a significantly large value of stand-off results in minimal heat flux from the flame and droplet evaporation rate. Therefore, the droplet diameter remains nearly unchanged and is considered a constant value at least in the duration of the droplet flight time for the respective run.

It is also to be noted that the bright yellow diffusion flame tip is identified when the local intensity of the luminous portion is significantly higher than the surrounding pixels in the flame image. To support this, the colored images show the luminous bright tip to have characteristic yellow color, suggesting the presence of a sooty fuel rich diffusion flame, as shown in Fig. 5 (*).

\section{DYNAMICS OF WAKE FLAME FOR $R e<120$}

\section{A. Wake flame dynamics and flame shape for $\psi<\mathbf{5 . 5}$}

For $\psi<5.5$ (regime II), due to smaller droplet diameter, a smaller flame is formed that exhibits lower fuel consumption rate. From the flame imaging, the edge-flame for $\psi>\mathbf{5 . 5}$ is observed to be almost four times more intense than $\psi<\mathbf{5 . 5}$. This also indicates higher fuel consumption rate for $\boldsymbol{\psi}>\mathbf{5 . 5}$ flames, which can be stabilized at higher flow velocities ${ }^{7}$ and can follow the droplet closely. Hence for $\psi<\mathbf{5 . 5}$, the flame stand-off $(\delta)$ continuously increases with increase in Reynolds number at a higher rate compared to $\boldsymbol{\psi}>\mathbf{5 . 5}$ as shown in Figs. 6 and 7(a). This increase in stand-off distance $(\delta)$ can lead to the dilution of the fuel stream. Continuous dilution of the fuel stream causes the transition from characteristic yellow diffusion flame 


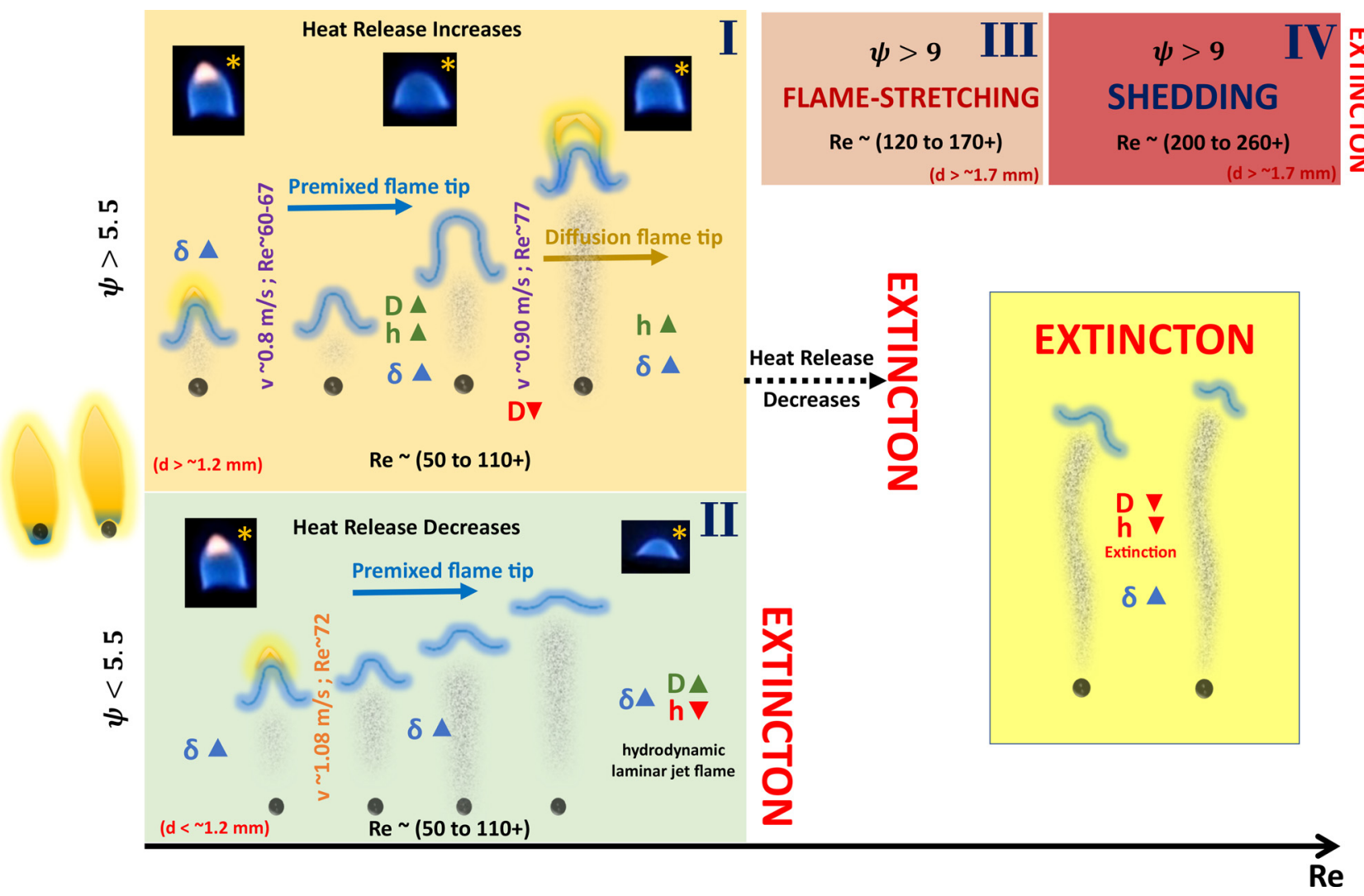

FIG. 5. The pictorial representation of the flame dynamics and characteristics at different non-dimensional parameter $(\psi)$ and Reynolds number $(R e)$. Here, $d$ represents droplet diameter, D represents flame width, $\delta$ represents flame stand-off distance from droplet, and h represents flame height. Regimes I and II in the range $R e<110$ show different flame topological transitions. For $\psi>9$, flame stretching and shedding occur at $R e>120$ and $R e>200$, respectively. The colored flame images in each regime (denoted by *) are the DSLR images showing the yellow tip taken in pendant mode with co-flow. Multimedia view: https://doi.org/10.1063/5.0073545.5

to a pale blue premixed flame at $R e \sim 70$, as depicted in Fig. 5. The higher rate of increase in $\delta$ with increase in Reynolds number implies a lower downward velocity of the flame. This results in lower relative velocity of the surrounding oxidizer with respect to the flame. Hence, the dilution effect of the jet expansion dominates, resulting in the mixture becoming progressively leaner with increase in $\delta$. Consequently, the flame intensity decreases temporally [see Fig. 2(a)].

During the flame transition to pale blue color when the excess fuel in the inner core of the flame is depleted, the yellow diffusion flame tip will disappear. It is reasonable to assume that the complete depletion of excess fuel in inner core occurs when the local equivalence ratio $(\phi)$ near the flame becomes $\phi<\mathbf{1}$. After this stage, as shown in Fig. 6(b), hydrodynamic laminar jet-like flow occurs where momentum diffuses, and the jet expands with axial propagation of the flow. ${ }^{5}$ This behavior is reflected in the flame shape as well, i.e., the flame height (h) decreases, and flame width (D) increases with increase in $\delta$, as depicted in Fig. 5.

\section{B. Wake flame dynamics and flame shape for $\psi>\mathbf{5 . 5}$}

The continuous increase in flame stand-off distance $(\delta)$ is supposed to render the mixture fuel-lean, resulting in a decrease in flame intensity. On the contrary, the opposite is observed to occur in regime I $(\psi>5.5)$, i.e., flame intensity starts to increase beyond $R e>60$, as shown in Fig. 5. This suggests that there is some mechanism through which the fuel flow rate is enhanced beyond $R e>60$, which makes the mixture fuel rich, thereby enhancing the flame intensity [see Fig. 2(b)]. It is to be noted that when the local equivalence ratio $(\phi)$ near the flame increases beyond $\phi>1$, the inner core becomes fuel rich due to excess fuel, and hence the pale blue flame transitions into a characteristic yellow diffusion flame. Even though the fuel stream already has undergone air entrainment, the addition of excess fuel to the mixture compensates for the dilution effect, resulting in yellow diffusion tip. The excess fuel in the inner fuel-rich wing $(\boldsymbol{\phi}>\mathbf{1})$ diffuses downstream to form a diffusion flame front [see Fig. 6(e)]. The increase in fuel flow rate into the flame is responsible for the increase in diffusion flame tip. ${ }^{41}$

Increase in fuel flow rate is caused by momentum diffusion from the surrounding air due to its relative motion with respect to the flame and fuel vapor field. The extent of momentum diffusion due to viscous effects becomes dominant as a result of the velocity gradient in order to reduce the relative velocity between the fluid layers. In other words, more the velocity gradient, higher the extent of momentum diffusion. As shown in Fig. 6, the flame size is larger in the case of $\boldsymbol{\psi}>\mathbf{5 . 5}$ (i.e., 


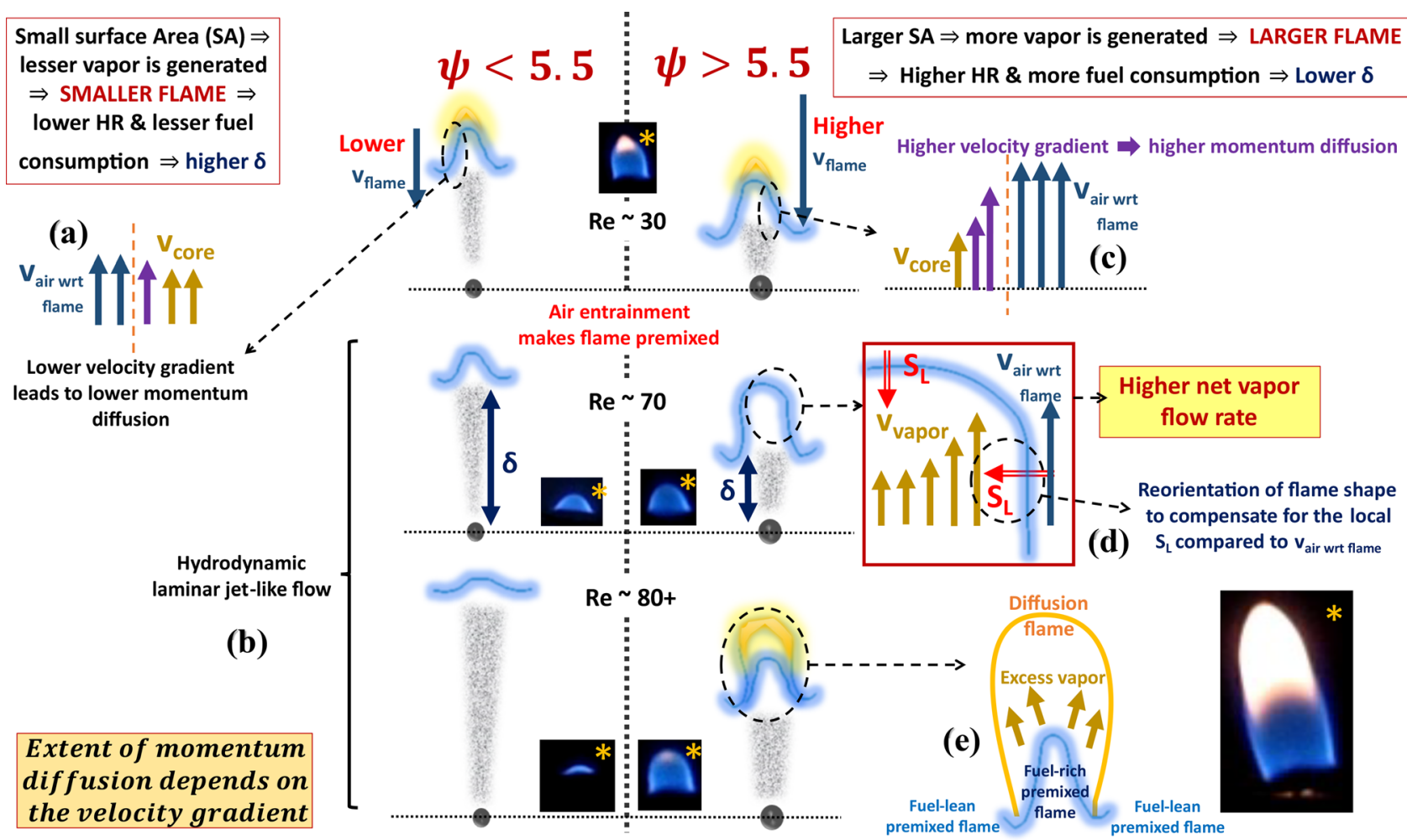

FIG. 6. The schematic of the mechanism of the flame dynamics for different non-dimensional parameter $(\psi)$ with increase in Reynolds number $(R e)$. The wake flame has been depicted at three different $R e$, i.e., three different vertical locations of droplet corresponding to both cases $\psi<\mathbf{5 . 5}$ and $\psi>\mathbf{5 . 5}$ (both at same vertical locations) on left side and right side of the central vertical dotted line, respectively. Here, $\delta$ represents flame stand-off distance from droplet, $v$ represents flow velocity, Re is Reynolds number, and $\psi$ is non-dimensional parameter. SA is surface area and HR is heat release. The colored flame images in each case (denoted by *) are the DSLR images showing the yellow tip taken in pendant mode with co-flow. [(a) and (c)] The momentum diffusion of air into the fuel vapor stream. (b) Hydrodynamic laminar jet like behavior for $\boldsymbol{\psi}<\mathbf{5 . 5}$. (d) Depiction of net increase in the vapor flow rate of fuel and the flame shape reorientation to compensate for higher flow velocity at the periphery. $S_{\mathrm{L}}$ is local flame propagation speed. (e) The schematic of a tribrachial flame or triple flame showing the fuel-lean and rich ends of premixed flame and the diffusion flame envelope. (Note that all the velocities shown in subfigures are in the reference frame of the flame.)

$\mathrm{d}>1.15 \mathrm{~mm}$ ) because of the higher fuel availability corresponding to the larger droplet surface. As a result, the stand-off distance $(\delta)$ is shorter due to the faster fuel consumption rate for larger $\psi$ when compared to $\psi<\mathbf{5 . 5}$; see Figs. 6 and 7 (a). This is also reflected in the more intense edge flame for regime I $(\psi>5.5)$ as consequence of higher fuel consumption rate. In both regimes I and II, $\delta$ increases with $R e$. The faster rate of increase in $\delta$ implies that the actual downward velocity of the flame is lower. This is also evident from (a)

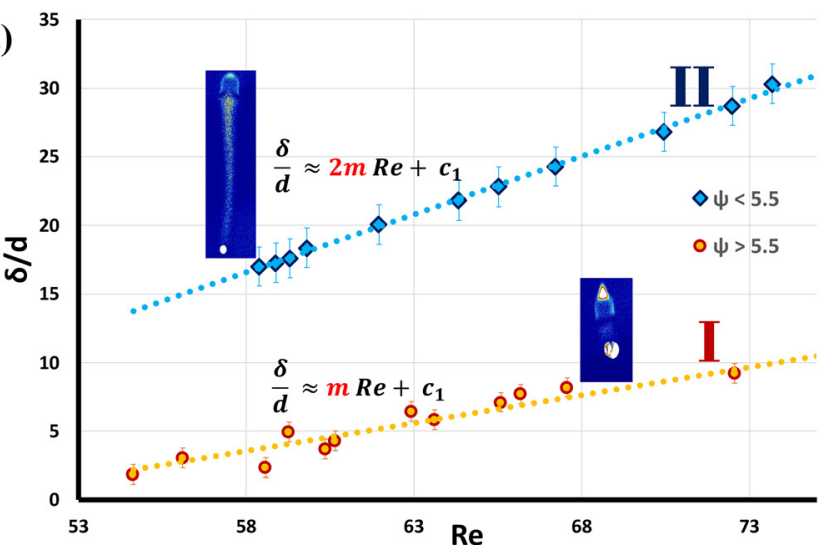

(b)

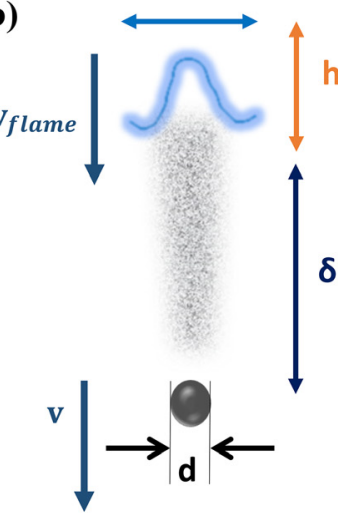

FIG. 7. (a) Plot showing change of normalized flame stand-off $(\delta / d)$ against Reynolds number $(R e)$. (b) The schematic showing different dimensions of the wake flame in the apt side of freely falling droplet. Here, $m$ and $c_{1}$ are constants of generalized line equation, $d$ represents droplet diameter, D represents flame width, $\delta$ represents flame stand-off distance from droplet, $\mathrm{h}$ represents flame height, and $v$ and $v_{\text {flame }}$ represent the falling velocity of the droplet and flame respectively with respect to ground. The error bars represent the standard error. 
experiments that for $\boldsymbol{\psi}<\mathbf{5 . 5}$, the flame exhibits a significantly lower downward velocity of descent $(<0.3 \mathrm{~m} / \mathrm{s})$ when compared to $\boldsymbol{\psi}>\mathbf{5 . 5}$ $(\sim 0.5 \mathrm{~m} / \mathrm{s})$, as shown in Figs. 6 and 7 (a). This implies that the relative flow velocity of the surrounding oxidizer with respect to the flame is faster in the case of $\boldsymbol{\psi}>\mathbf{5 . 5}$.

It is reasonable to assume that the velocity in the core of the fuel stream is primarily due to buoyancy and diffusion for both regimes I and II $(\sim 0.1-0.2 \mathrm{~m} / \mathrm{s})$, excluding the surrounding relative flow effects. The velocity gradient is significantly higher in the case of $\psi>\mathbf{5 . 5}$ (regime I) when compared to $\boldsymbol{\psi}<\mathbf{5 . 5}$ (regime II) as shown in Figs. 6(c) and 6(a). Hence, the extent of momentum diffusion is more for $\psi>5.5$ compared to regime II, resulting in higher vapor velocity, which increases the net fuel vapor flow rate into the flame, as depicted in Figs. 6(c) and 6(d). This phenomenon can explain the existence of a higher amount of fuel beyond $R e>60$, which leads to an increase in flame intensity in regime I $(\psi>5.5)$.

The effect of species diffusion is relatively insignificant as $\vartheta_{a}>\mathfrak{D}_{a-D D}$ by one order and hence is neglected for simplicity in scaling analysis. The data also indicate reorientation of the flame shape around $R e \sim 70$, as shown in Fig. 6(d). At $R e \sim 70$, the local outer flow velocity with respect to flame $(\sim 0.5 \mathrm{~m} / \mathrm{s})$ is relatively higher than the flame propagation speed $\sim(0.4 \mathrm{~m} / \mathrm{s})$ for leaner mixture at the periphery. ${ }^{43}$ Hence, the flame shape and its orientation are self-adjusted to remain stabilized which further suggests the increase in local flow velocity in the vapor stream due to momentum diffusion. This readjustment is achieved in such a way that the normal component of fuel flow to the flame surface will balance the local flame propagation speed, ${ }^{8}$ as shown in Fig. 6(d).

\section{RESULTS AND DISCUSSION}

\section{A. Estimation of transition between premixed and diffusion flame using equivalence ratio $(\phi)$}

So far, it has been established that the wake flame dynamics are predominantly influenced by the fuel flow rate variation modulated by momentum diffusion due to the relative motion of the surrounding oxidizer and the dilution effect. The value of equivalence ratio $(\boldsymbol{\phi})$ needs to be evaluated to quantitatively estimate when the flame transitions to/from the diffusion flame using a theoretical formulation. The schematic of the dimensions that describe the flame shape, position, and velocity are shown in Fig. 7(b). In order to calculate the equivalence ratio $(\boldsymbol{\phi})$, the mass flow rate of fuel $\left(\dot{\boldsymbol{m}}_{\boldsymbol{f}}\right)$ and oxidizer $\left(\dot{\boldsymbol{m}}_{\boldsymbol{a}}\right)$ should be estimated, which is given as

$$
\phi=\frac{\dot{\boldsymbol{m}}_{f}}{\dot{\boldsymbol{m}}_{\boldsymbol{a}}}=\frac{\rho_{f} \dot{\boldsymbol{Q}}_{f}}{\rho_{a} \dot{\boldsymbol{Q}}_{\boldsymbol{a}}},
$$

where $\dot{\boldsymbol{Q}}_{f}, \dot{\boldsymbol{Q}}_{\boldsymbol{a}}$ and $\rho_{f}, \rho_{a}$ are the flow rates and densities of fuel and air, respectively.

$\boldsymbol{Q}_{a}$ can be estimated by adding up the air entrainment into the fuel stream for overall length $[\delta+h]$. This is computed based on the radially inward velocity, which comprises of $\mathrm{v}_{a, \text { advection, } D}$ and $\mathrm{v}_{a \text {, diffusion, } D}$ scaled as follows:

$$
\begin{aligned}
\mathrm{v}_{a, \text { advection }, D} & \sim \mathrm{v} \frac{D}{\delta}, \\
\mathrm{v}_{a, \text { diffusion }, D} & \sim \frac{\mathcal{D}}{D},
\end{aligned}
$$

where $\mathbf{v}$ is the axial velocity scale of air due to droplet motion. The average value $\frac{d+D}{2}$ is used for computing $\dot{\boldsymbol{Q}}_{\boldsymbol{a}}$ which is given by the following equation:

$$
\dot{Q}_{a} \sim \pi\left(\frac{d+D}{2}\right)[\delta+h]\left(\mathrm{v}_{a, \text { advection }, D}+\mathrm{v}_{a, \text { diffusion }, D}\right) .
$$

The estimation of $\dot{Q}_{f}$ should include the effect of momentum diffusion from the surrounding air, which depends on the velocity gradient. Enhanced momentum diffusion occurs when the difference between the velocity of fuel stream and air with respect to the flame is large, which results in increase in vapor mixture velocity, leading to the overall increase in $\dot{\boldsymbol{Q}}_{f}$, as shown in Figs. 6(c) and 6(d). This effect can be incorporated using the following equation:

$$
\mathrm{v}_{\text {vapor, avg }} \sim \frac{\boldsymbol{\alpha} \mathrm{v}_{\text {flame }}+\mathrm{v}_{\text {core }}}{\boldsymbol{\alpha}+1},
$$

where the parameter $\boldsymbol{\alpha} \sim \frac{\mathrm{v}_{\text {flame }}}{\mathrm{v}_{\text {ore }}}$ represents the extent of momentum diffusion into the fuel stream from the relative motion of the oxidizer. $\mathrm{v}_{\text {flame }}$ is the velocity with which the flame moves with respect to ground which is equal to the relative velocity of the airflow imposed on the flame. $\mathrm{v}_{\text {core }}$ is the velocity at the core of the fuel stream, which is driven by the buoyancy and diffusion that can be scaled as shown in the following equation:

$$
\mathrm{v}_{\text {core }} \sim\left(\mathrm{v}_{\text {buoyancy,d }}+\mathrm{v}_{D D, \text { diffusion }, d}\right),
$$

where both $\mathrm{v}_{D D \text {,buoyancy,d }}$ and $\mathrm{v}_{D D \text {,diffusion,d }}$ are estimated using droplet diameter as the length scale. Thus, using this velocity scale of the fuel stream, the net fuel flow rate is estimated as

$$
\dot{Q}_{f} \sim \mathrm{k}_{1}\left[\frac{\pi D^{2}}{4} \mathrm{v}_{\text {vapor, avg }}\right],
$$

where $k_{1}$ is constant. Finally, the equivalence ratio can be estimated by substituting Eqs. (6)-(11) in Eq. (5). The results are shown in the Fig. 8 , where the equivalence ratio $(\boldsymbol{\phi})$ is plotted against Reynolds number $(R e)$.

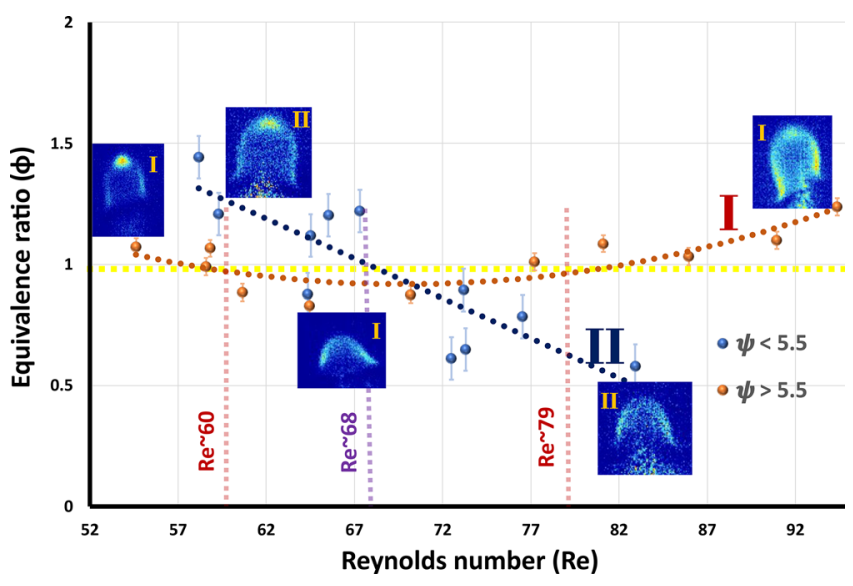

FIG. 8. Equivalence ratio $(\phi)$ is plotted against Reynolds number $(R e)$ for regimes I and II. The flame images for corresponding regimes are indicated in the plot. The error bars represent the standard error. 
In Fig. 8, there is a clear decreasing trend of $\phi$ with Reynolds number for $\psi<\mathbf{5 . 5}$ (regime II). Interestingly, the value of $\boldsymbol{\phi}$ falls below 1 around $R e \sim 68$. This suggests that excess fuel present during $R e<68$ got depleted, resulting in the disappearance of the characteristic yellow diffusion flame tip. Beyond $R e>68$, the flame changes into pale blue color, suggesting no soot formation, complete combustion, and premixedness. On the other hand, in regime $I(\psi>5.5)$, first decreasing and subsequent increasing trend of $\phi$ is observed from Fig. 8. The value of $\boldsymbol{\phi}$ is initially greater than 1 , before sliding below 1 around $R e>60$. However, it starts to increase subsequently, crossing $\phi>1$ around $R e>79$. This suggests that the initial characteristic yellow diffusion flame tip should disappear around $R e>60$, transitioning to completely premixed flame, and the yellow tip should reappear beyond $\operatorname{Re}>79$. Both these predictions based on $\phi$, regarding the transition between diffusion flame and complete premixed flame, are in good agreement with the experimental observations with less than $\pm 10 \%$ uncertainty, as depicted in Figs. 8 and 5. All the trends observed have been properly captured by the theoretical formulation explaining the flame dynamics qualitatively and quantitatively for both the regimes I and II. For significantly higher droplet diameters, i.e., for $\psi>9$ (regimes III and IV), the transition between completely premixed to diffusion flame is observed similar to $\psi>\mathbf{5 . 5}$ i.e., regime I [see Fig. 3(a)]. Moreover, for $R e<(130-150)$, the proposed theoretical formulation agrees with experiments in this $\psi>\mathbf{9}$ regime as well.

\section{B. Wake flame oscillations at high Reynolds number (Re $>130)$}

\section{Vortex shedding in droplet wake}

At higher $R e>(130-150)$, the onset of asymmetric detachment of vortices in the droplet wake has been observed. It is specifically noted that since the flame is located in the wake, at a distance of more than 10 times the droplet diameter, the temperature is not high enough near the droplet surface. Low temperature leads to lower value of kinematic viscosity in the wake leading to reduced viscous dissipation of vorticity. In the vorticity transport equation [Eq. (12)], the first term on the right-hand side represents vortex stretching which can have a non-zero value because of BVK instability. The second term is the gas expansion term which will not have a significant value due to the incompressible flow assumption at low temperature and for the present range of Reynolds number. The third term is the vorticity generation term due to the baroclinic torque, which arises due to the density gradient near the shear layer. The last term, which accounts for the viscous effects, has a relatively low value when compared to the other terms due to lower temperature. ${ }^{39}$ All the terms cumulatively indicate the presence of a significant non-zero value of vorticity present in the wake of the droplet, which is responsible for the aforementioned asymmetric vortex detachment at high Reynolds number,

$$
\frac{D \omega}{D t}=(\omega \cdot \nabla) \mathrm{v}-\omega(\nabla \cdot \mathrm{v})+\frac{\nabla \rho \times \nabla p}{\rho^{2}}+\vartheta \nabla^{2} \omega .
$$

The temperature in the wake of the droplet is assumed to be around the wet-bulb temperature of dodecane.

These vortices have to travel all the way from the droplet and interact with the wake flame, as depicted in Fig. 9(a). It is known that the frequency of alternate shedding of Von Karman vortex street induced by BVK instability can be estimated using Strouhal number,
$S t \sim f(R e) .{ }^{44}$ Hence, the vortex shedding frequency can be calculated using the relation $f_{B V K} \sim S t(\mathrm{v} / d)$. The wake flame response frequency is experimentally observed to be equal to half of BVK vortex shedding frequency $\left(f_{B V K}\right)$ and is within $\pm(2-4) \%$ of uncertainty, as shown in Figs. 4(c) and 4(d) (boxed information) for the corresponding experimental runs. Hence, it can be concluded that the vortex shedding in the wake is induced by the BVK instability. The wake flame is observed to respond each time as it encounters these vortices i.e., half cycle of BVK shedding. During the interaction between aforementioned vortex and wake flame, a secondary vortex shedding event occurs at the flame [see Fig. 9(a)]. The flame stretching/pinch-off or bulk shedding events occur depending on the strength of these vortices which depends on the velocity scale $(R e)$. The vapor trail emanating from the droplet offers qualitative insights into the flow features behind the droplet. The Von Karman vortex street like behavior of the wake is reflected in the deformation of the vapor trail as shown in Figs. 9(a) and 9(b). Each inflexion point in the vapor trail can be surmised to be the location where each vortex eye of the vortex street is located. These vortices travel downstream, and the wake flame is observed to interact with each of the vortex centers along its way upstream, thereby undergoing stretching/shedding. These puffing/shedding events are reflected in the momentary spikes in flame intensity [see Figs. 4(c) and $4(\mathrm{~d})$ ].

The entire phenomenon of droplet wake flame interaction at high Reynolds number $(R e)$ is observed to exhibit two types of vortex shedding. One is due to BVK instability at high Reynolds number that occurs both with and without the presence of wake flame, which is just a flow phenomenon. However, only in the presence of wake flame, these Bernard-Von Karman (BVK) induced vortices interact with the wake flame, resulting in secondary vortex shedding due to circulation build-up along the flame length. This ultimately results in rigorous flame puffing/stretching or pinch-off/shedding events. The flame puffing/shedding events only occur at high Reynolds number where wake is characterized by the BVK vortex street and do not occur at lower Reynolds number $(R e)$ when the droplet wake is non-oscillating. However, even at high Reynolds number, the BVK vortex shedding would not occur if the wake flame is located near the droplet at the rear stagnation point. ${ }^{39}$ The higher wake temperature leads to dissipation of vorticity [dominance of last term in Eq. (12)]. Hence, the flame will be relatively quiescent in the absence of incoming BVK vortices.

\section{Effect of BVK vortex shedding and correlation with flame shedding height}

Experimental observations show that, in the range $R e$ $\sim(150-170)$ i.e., regime III, the flame starts to stretch, finally leading to pinch-off [Fig. 2(c)]. In regime IV ( $R e>200)$, the flame undergoes violent bulk shedding (Fig. 9), followed by extinction phase due to rapid fuel consumption. All these phenomena can be seen in the $\psi>\mathbf{9}$ regime of Fig. 3(a) but not for $\boldsymbol{\psi}>\mathbf{5 . 5}$ as the flame is sustained till higher $R e>120$ for $\boldsymbol{\psi}>\mathbf{9}$, thus delaying extinction due to the higher fuel availability. These puffing/stretching or shedding events lead to substantial increase in the flame size, especially the luminous yellow portion, which account for this momentary increase in flame intensity as shown in Figs. 4(c) and 4(d). The wake flame responds at half the BVK vortex shedding frequency [see Figs. 4(c) and 4(d) (boxed information)] as it interacts with each vortex (half cycle) on 

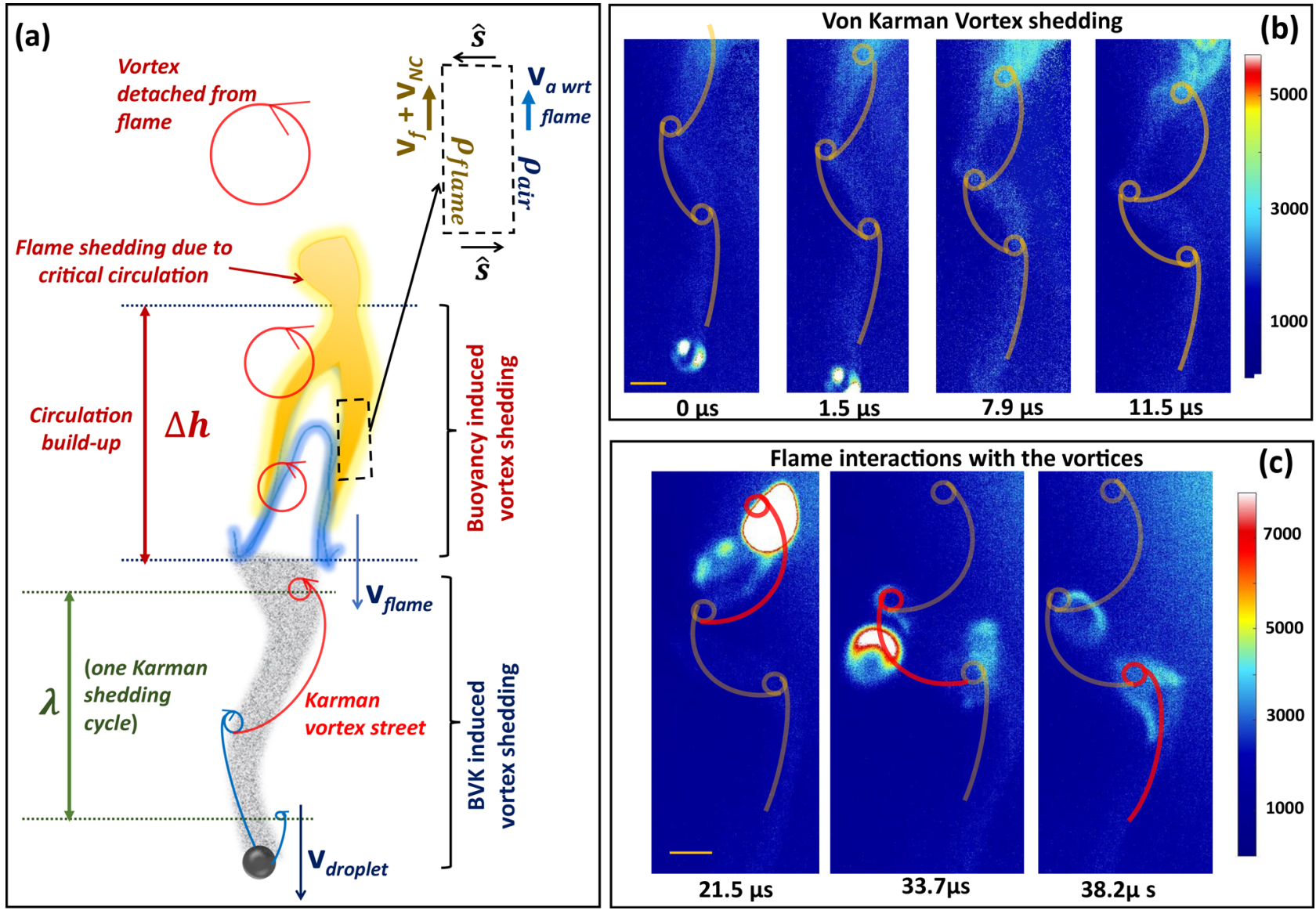

FIG. 9. (a) Schematic representation of Bernard-Von Karman (BVK) vortex interaction with the wake flame at high $R e \sim 200$ for $\boldsymbol{\psi}>\mathbf{9}$. The red circles indicate the circulation build-up of the BVK vortex center that is interacting with the flame. The two types of vortex-shedding phenomena, BVK-induced and buoyancy-induced shedding, are depicted. The velocity scales and material contour at the flame shear layer are shown in top right corner. $\lambda$ represents the single cycle of Von Karman vortex shedding, and $\Delta \mathrm{h}$ indicates shedding height and $\hat{s}$ represents the contour. $v_{f}$ represents flow velocity on the flame side, $v_{N C}$ represents buoyant natural convection velocity scale, and $v_{a}$ represents the relative velocity of air. (b) The time series of Von Karman vortex street like behavior of the vapor trail and (c) the time series of flame interaction with the vortices causing flame puffing/shedding events in a freely falling burning droplet in regimes III and IV. The Karman vortex street is depicted overlaying the images for reference. The location of vortex-flame interaction is highlighted using red colored circle. The color bar indicates the intensity in the respective images. All the scale bars represent $2 \mathrm{~mm}$. Multimedia view: https://doi.org/10.1063/5.0073545.6

either side of vortex street, as shown in Figs. 9(a) and 9(c). While the wake flame responds to these vortices, i.e., external forcing, the buoyancy-induced circulation build-up occurs along the flame, followed by detachment of these vortices from the flame, resulting in flame shedding events.

The flame shedding due to buoyancy-induced vortex roll-up is characterized using the shedding height (h). Kinematic formulation by Cetegen et al. estimated ${ }^{13}$ the scaling for flame puffing/shedding frequency $\left(f_{\text {shedding }}\right)$ in the case of pool fires and plumes as shown in Eq. (13) in terms of Richardson number ( $R i)$ number,

$$
f_{\text {flame }}=k \sqrt{\frac{g}{D_{o}}}\left[\left(1+\frac{1}{R i}\right)^{1 / 2}-\frac{1}{\sqrt{R i}}\right]^{-1},
$$

where constant $k$ depends on densities of gases and $D_{o}$ is the nozzle diameter.
The wake flame for higher Reynolds number for the $\boldsymbol{\psi}>\mathbf{9}$ regime is observed to have a significantly large diffusion flame envelope that arises from the $\boldsymbol{\phi}=\mathbf{1}$ point as shown in Fig. 6(e). Additionally, a larger value of $\psi$ suggests that the buoyancy effects are significant corresponding to high Richardson number limit. Flame shedding occurs when the buoyant flame interacts with external forcing and shedding height $(\mathrm{h})$ is the associated convective length scale. ${ }^{12}$ Thus, the shedding height $(\mathrm{h})$ is the unique characteristic length scale correlated with flame response frequency. Using the relation in Eq. (13) for large Richardson number limit $(R i \gg 1)$, the following simplified relation is obtained for flame shedding frequency:

$$
f_{\text {flame }} \sim \sqrt{\frac{g}{h}}
$$

Under external forcing due to BVK induced vortex shedding, the wake flame responds while interacting with the vortices, leading to flame 
shedding. Hence, the flame response frequency $\left(f_{\text {flame }}\right)$ is only dependent on the BVK vortex shedding phenomenon. However, the local flame oscillation characteristics are governed by the buoyancy-induced vortex shedding near the flame, coupled with the incoming BVK vortices. Using Eq. (14), the shedding height (h) associated with flame oscillation at the flame response frequency can be estimated even for the droplet wake flame. Figure 10(a) shows the experimentally observed shedding heights (h) plotted against their corresponding flame oscillation frequency $\left(f_{\text {flame }}\right)$. The scaling offered by Eq. (14) is represented as a solid blue line and is observed to be in very good agreement with the experimental data within $\pm 10 \%-12 \%$ of uncertainty, as shown in Fig. 10(a). Hence, the wake flame shedding mechanism due to the interaction of the flame with vortex street at high Reynolds number can be explained using the scaling presented in Eq. (14). It shows that a unique correlation exists between the local buoyancy-induced vortex detachment height along the flame (h) and the frequency/timescale of the flame response governed by the BVK vortex frequency. In other words, in the presence of droplet wake flame at high $R e$, the momentumdominated BVK vortices will translate into the local buoyancyinduced vortex detachment phenomenon near the flame causing flame shedding. It is interesting to note that flame shedding surprisingly turns out to be predominantly buoyancy-driven following the same scaling as buoyant flame flickering [see Fig. 10(a)] even though the momentum-dominated vortex street triggered it.

The effect of flow velocity around the flame on the flame shedding has to be analyzed. From the experiments, the shedding height of the flame is observed to have a decreasing trend with the increase in Reynolds number, as shown in Fig. 10(b). This can be explained using vortex roll-up and critical circulation mechanism where the disturbances get aggravated due to buoyancy-induced instability at the flame, while the vortices interact with the flame. The vortex roll-up leads to the accumulation of circulation inside the system, as shown in Fig. 9(a) which has to be theoretically estimated. Using a similar approach as Xia et al. using vorticity transport Eq. (12) near the flame, ${ }^{15}$ the circulation build-up equation can be determined, given by Eq. (15). In the RHS of Eq. (12), the first term can be neglected since flow can be

(a)

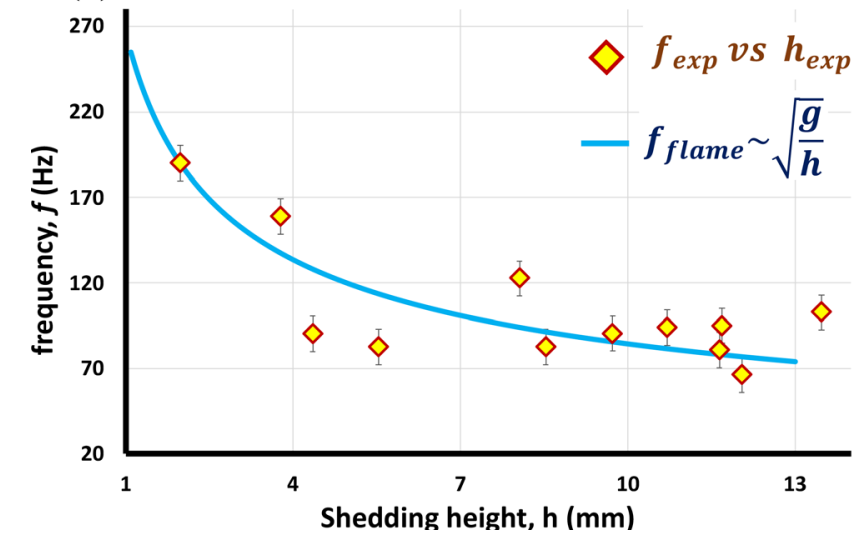

considered non-swirling, axisymmetric near the flame, and the second term is negligible due to incompressible assumption. The last term in Eq. (12) represents the vorticity-dissipation term which can be neglected compared to the baroclinic term (third term) due to high temperature of at the flame. ${ }^{12}$ Thus, obtaining Eq. (15), the temporal variation of circulation is found out to be the combination of gravity generated vorticity term and the initial circulation strength fed into the flame by the virtue of initial velocity scale before the interaction,

$$
\frac{d \Gamma}{d t}=\rho_{a} g\left(\frac{1}{\rho_{a}}-\frac{1}{\rho_{f}}\right) \Delta h+\frac{d \Gamma_{\text {initial }}}{d t} .
$$

The initial velocity scale across the shear layer is considered, as shown in the top right corner of Fig. 9(a). Substituting these initial velocity scales in initial circulation term [as shown in Eq. (16)] of Eq. (15) gives Eq. (17). It is to be noted that the initial velocity scales are considered in the reference frame of the flame,

$$
\begin{gathered}
\frac{d \Gamma_{\text {initial }}}{d t}=\left(\mathrm{v}_{\text {flame }}\right)^{2}-\left(\mathrm{v}_{N C}+\mathrm{v}_{f}\right)^{2} \\
\frac{d \Gamma}{d t}=\rho_{a} g\left(\frac{1}{\rho_{a}}-\frac{1}{\rho_{f}}\right) \Delta h+\left(\mathrm{v}_{\text {flame }}\right)^{2}-\left(\mathrm{v}_{N C}+\mathrm{v}_{f}\right)^{2} .
\end{gathered}
$$

The vortices roll-up along the flame and detach, resulting in flame shedding when the circulation build-up reaches a critical value. Equation (17) has to be integrated over a shedding cycle using the same scaling $\left(t_{\text {shedding }} \sim \sqrt{h / g}\right)$ to ascertain the critical circulation. However, the value of $v_{f}$ (flow velocity on the flame side) cannot be found accurately due to experimental limitations. Nevertheless, Eq. (17) can be used to characterize the flame shedding phenomenon.

The first term in Eq. (17) is totally buoyancy-driven and hence will remain unchanged even with change in surrounding velocity or initial velocity conditions. However, the second term is a direct function of relative velocity of surrounding air with respect to flame $\left(\mathrm{v}_{\text {flame }}\right)$. The third term is a function of $\mathrm{v}_{N C}$ (buoyant velocity scale for natural convection) and $\mathrm{v}_{f}$ (flow velocity on the flame side). $\mathrm{v}_{N C}$ is

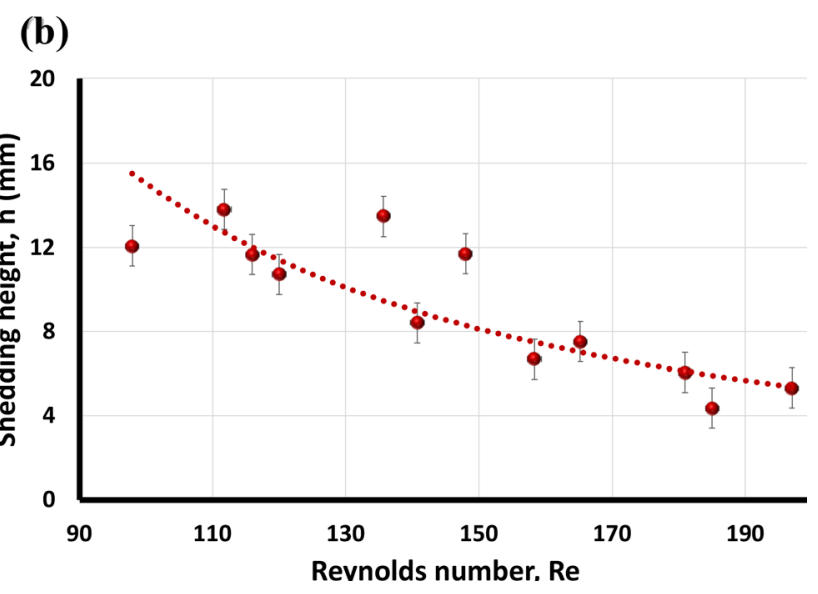

FIG. 10. (a) Plot of shedding frequency ( $f$ ) against shedding height ( $h$ )-points correspond to experimental data and blue line correspond to the theoretical scaling; (b) plot showing the experimentally observed decreasing trend of pinch-off/shedding height (h) with increasing Reynolds number as predicted by circulation build-up mechanism. The error bars represent the standard error. 
independent of initial velocity conditions, but the flow velocity at the flame $\left(\mathrm{v}_{f}\right)$ will depend on $\mathrm{v}_{\text {vapor }}$ which is a function of $\mathrm{v}_{\text {flame }}$ [see Eq. (9)]. However, in this regime $(\boldsymbol{\psi}>\mathbf{9})$, since the flame is buoyancy dominant due to high $R i \gg 1$ that results in the higher value of $\mathrm{v}_{\text {core }}$, the influence of $\mathrm{v}_{\text {flame }}$ on $\mathrm{v}_{\text {vapor }}$ will be minimal [smaller value of $\boldsymbol{\alpha}$ in Eq. (9)] as the velocity gradient across shear layer will be relatively less. This is also reflected by the high value of $\psi$ in this regime that indicates the relative dominance of the buoyancy and minimal effect of momentum diffusion or entrainment on the inner flow. This makes $\mathrm{v}_{f}$ less sensitive to the surrounding velocity $\left(\mathrm{v}_{\text {flame }}\right)$ or the initial velocity conditions in this regime. Hence, in Eq. (17), the second term $\left(\mathrm{v}_{\text {flame }}\right)^{2}$ will be more sensitive to the initial velocity conditions or surrounding velocity when compared to the third term $\left(\mathrm{v}_{N C}+\mathrm{v}_{f}\right)^{2}$ which has relatively weaker dependence on the surrounding velocity $\left(\mathrm{v}_{\text {flame }}\right)$. This implies that with increase in Reynolds number of the surrounding flow, i.e., the initial velocity scale, the second term in Eq. (17) will increase more when compared to third term. Hence, the LHS, i.e., the net rate of circulation build-up, will increase with Reynolds number.

Hence, it has been established that at higher Reynolds number, circulation build-up in the system will be faster due to the initial velocity scale. When this circulation build-up reaches a critical value, the flame shedding occurs. Thus, for high Reynolds number, the critical circulation is reached faster over a shorter shedding height, as shown in Fig. 10(b). This can be observed from Fig. 4 (†), [Figs. 2(c) and 9] which illustrates that the shedding height is shorter for regime IV, with higher Reynolds number $(R e>200)$ compared to regime III $(R e$ $\sim 150-170$ ). Thus, we can infer that the circulation build-up mechanism indicated by Eq. (17) agrees with the decreasing trend of shedding height (h) with increase in Reynolds number, which is observed experimentally [see Fig. 10(b)]. Hence, the vortex roll-up and circulation build-up mechanism is applicable for the flame shedding events in the droplet wake flame at high Reynolds number even when the flame shedding is a consequence of the momentum induced Bernard-Von Karman vortex shedding.

\section{SUMMARY AND CONCLUSIONS}

The wake flame dynamics has been studied in the current study for a freely falling burning droplet for a low vapor pressure fuel, dodecane. The fully enveloped diffusion flame around a droplet transitions into a wake flame as the droplet accelerates under gravity due to forward extinction. The wake flame is observed to be stabilized at the end of the fuel vapor trail emanating from the droplet. With an increase in Reynolds number, more fuel gets accumulated in the droplet wake, elongating the vapor trail present downstream of the droplet. The wake flame anchors itself to the end of the vapor trail and follows it as the droplet falls. The wake flame is observed to be similar to a tribrachial laminar lifted flame. The different regimes have been identified based on the wake flame behavior for different droplet diameters and Reynolds number $(R e)$. A new non-dimensional parameter $(\psi)$ has been proposed to account for the local balance between buoyancy and momentum diffusion. This modifies the fuel availability at the flame that governs the flame topological evolution with increasing Reynolds number. The flame intensity is observed to show an increasing and decreasing trend with an increase in Reynolds number, for $\psi>\mathbf{5 . 5}$ and $\psi<5.5$, respectively, accompanied by the flame transition from premixed to diffusion flame when the flame intensity increases and vice versa for $R e>60$. This change in flame intensity with Reynolds number is attributed to the change in fuel mixture fraction at the flame. A theoretical formulation has been proposed, which suggests that the fuel availability at the flame depends on the fuel flow rate in the vapor stream. The momentum diffusion from the surrounding gas phase dictated by the velocity gradient across the shear layer influences the fuel flow rate. This is found to be in good agreement with the experimental data in predicting the flame transitions. It also accounts for the effect of droplet diameter on the flame behavior both qualitatively and quantitatively with good precision. The non-dimensional parameter $(\psi)$ indicates the tendency of the transition to diffusion flame for the corresponding droplet size.

Furthermore, flame shedding/pinch-off events occur for $\boldsymbol{\psi}>\mathbf{9}$ at higher Reynolds number. The flame puffing/shedding or pinch-off events resulting in temporary spikes in the flame intensity are caused by the interaction of wake flame with the Von Karman vortex street. This is evident as the shedding frequency is observed to be half of the BVK vortex shedding frequency as the flame interacts with each Bernard-Von Karman vortex eye on either side of the vortex street. The shedding height $(\mathrm{h})$ is used to characterize the flame shedding. Interestingly, the shedding height reduces with increase in frequency and is in good agreement with the theoretical scaling of buoyant flickering $f_{\text {flame }} \sim \sqrt{\boldsymbol{g} / \boldsymbol{h}}$, even for the droplet wake flame involving high velocity scales. The shedding height is also observed to decrease with an increase in Reynolds number. This is because the buoyancyinduced circulation build-up in the system is faster and reaches the critical value at shorter heights due to higher flow velocity scales. Hence, it can be inferred that for high Reynolds number, the vortex roll-up and critical circulation mechanism is responsible for flame puffing/shedding events in the case of the droplet wake flame as well. The parameter $(\psi)$ has given insights into the interplay between buoyancy and momentum diffusion even in a high Reynolds number regime, which affects the flame shedding phenomenon.

\section{ACKNOWLEDGMENTS}

The authors are thankful to Swarnajayanti Fellowship Scheme, DRDO Chair Professor funding, for financial support.

\section{AUTHOR DECLARATIONS Conflict of Interest}

The authors report no conflict of interest.

\section{DATA AVAILABILITY}

The data that support the findings of this study are available from the corresponding author upon reasonable request.

\section{REFERENCES}

${ }^{1}$ C. K. Law, "Recent advances in droplet vaporization and combustion," Prog. Energy Combust. Sci. 8, 171-201 (1982).

${ }^{2}$ K. N. Kim, S. H. Won, and S. H. Chung, "Characteristics of laminar lifted flames in coflow jets with initial temperature variation," Proc. Combust. Inst. 31, 947-954 (2007).

${ }^{3}$ H. Phillips, "Flame in a buoyant methane layer," Symp. Combust. 10, 1277-1283 (1965).

${ }^{4}$ J. Buckmaster and N. Matalon, "Anomalous Lewis number effects in tribrachial flames,” Symp. Combust. 22, 1527-1535 (1989).

${ }^{5}$ S. H. Chung and B. J. Lee, "On the characteristics of laminar lifted flames in a non-premixed jet,” Combust. Flame 86, 62-72 (1991). 
${ }^{6} \mathrm{~S}$. Ghosal and L. Vervisch, "Theoretical and numerical study of a symmetrical triple flame using the parabolic flame path approximation,” J. Fluid Mech. 415, 227-260 (2000).

${ }^{7}$ M. Balasubramaniyan, A. Kushwaha, Y. Guan, J. Feng, P. Liu, V. Gupta, and L. K. B. Li, "Global hydrodynamic instability and blowoff dynamics of a bluffbody stabilized lean-premixed flame," Phys. Fluids 33, 034103 (2021).

${ }^{8}$ N. P. Sapkal, "Role of chemiluminescence and radius of curvature in the stabilization of methane/helium lifted flames," Int. J. Heat Technol. 36, 1249-1255 (2018).

${ }^{9}$ X. Qin, I. K. Puri, and S. K. Aggarwal, "Characteristics of lifted triple flames stabilized in the near field of a partially premixed axisymmetric jet," Proc. Combust. Inst. 29, 1565-1572 (2002).

${ }^{10}$ N. I. Kim, U. D. Lee, and H. D. Shin, "Extinction of a premixed flame by a large variation in axial velocity," Combust. Flame 136, 467-480 (2004).

${ }^{11}$ L. Muniz and M. G. Mungal, "Instantaneous flame-stabilization velocities in lifted-Jet diffusion flames,” Combust. Flame 111, 16-31 (1997).

${ }^{12}$ K. Pandey, S. Basu, B. Krishan, and V. Gautham, "Dynamic self-tuning, flickering and shedding in buoyant droplet diffusion flames under acoustic excitation," Proc. Combust. Inst. 38, 3141-3149 (2021).

${ }^{13}$ B. M. Cetegen and T. A. Ahmed, "Experiments on the periodic instability of buoyant plumes and pool fires," Combust. Flame 93, 157-184 (1993).

${ }^{14}$ D. Moreno-Boza, W. Coenen, A. Sevilla, J. Carpio, A. L. Sánchez, and A. Liñán, "Diffusion-flame flickering as a hydrodynamic global mode," J. Fluid Mech. 798, 997-1014 (2016).

${ }^{15} \mathrm{X}$. Xia and P. Zhang, "A vortex-dynamical scaling theory for flickering buoyant diffusion flames,” J. Fluid Mech. 855, 1156-1169 (2018).

${ }^{16}$ S. Kumagai, T. Sakai, and S. Okajima, "Combustion of free fuel droplets in a freely falling chamber,” Symp. Combust. 13, 779-785 (1971).

${ }^{17}$ P. M. Guerieri, S. DeCarlo, B. Eichhorn, T. Connell, R. A. Yetter, X. Tang, Z. Hicks, K. H. Bowen, and M. R. Zachariah, "Molecular aluminum additive for burn enhancement of hydrocarbon fuels," J. Phys. Chem. A 119, 11084-11093 (2015).

${ }^{18}$ P. M. Guerieri, J. B. DeLisio, and M. R. Zachariah, "Nanoaluminum/nitrocellulose microparticle additive for burn enhancement of liquid fuels," Combust. Flame 176, 220-228 (2017).

${ }^{19} \mathrm{~A}$. Makino and H. Fukada, "Ignition and combustion of a falling, single sodium droplet," Proc. Combust. Inst. 30, 2047-2054 (2005).

${ }^{20}$ C. H. Wang, X. Q. Liu, and C. K. Law, "Combustion and microexplosion of freely falling multicomponent droplets,” Combust. Flame 56, 175-197 (1984).

${ }^{21}$ C. H. Wang, S. Y. Fu, L. J. Kung, and C. K. Law, "Combustion and microexplosion of collision-merged methanol/alkane droplets," Proc. Combust. Inst. 30, 1965-1972 (2005).

${ }^{22}$ C. K. Law and F. A. Williams, "Kinetics and convection in the combustion of alkane droplets," Combust. Flame 19, 393-405 (1972).

${ }^{23}$ K. Meng, K. Han, F. Li, L. Bao, C. Wang, and Q. Lin, "Study on combustion and micro-explosion characteristics of biodiesel and ethanol mixture droplets under different oxygen concentrations and temperatures," Phys. Fluids 33, 052003 (2021).

${ }^{24} \mathrm{~K}$. Pandey, K. Chattopadhyay, and S. Basu, "Combustion dynamics of low vapour pressure nanofuel droplets," Phys. Fluids 29(7), 074102 (2017).
${ }^{25} \mathrm{~K}$. Pandey and S. Basu, "How boiling happens in nanofuel droplets," Phys. Fluids 30, 107103 (2018).

${ }^{26}$ D. C. K. Rao, S. Karmakar, and S. Basu, "Bubble dynamics and atomization mechanisms in burning multi-component droplets,” Phys. Fluids 30, 067101 (2018).

${ }^{27} \mathrm{G}$. Wu, W. A. Sirignano, and F. A. Williams, "Simulation of transient convective burning of an n-octane droplet using a four-step reduced mechanism," Combust. Flame 158, 1171-1180 (2011).

${ }^{28} \mathrm{~L}$. W. Huang and C. H. Chen, "Single droplet combustion in a gravitational environment," Wärme- Stoffübertrag. 29, 415-423 (1994).

${ }^{29}$ W. Wu, C. K. Law, and A. C. Fernandez-pello, "A unified criteria for the convective extinction of fuel particles," Combust. Flame 44, 113-124 (1982).

${ }^{30} \mathrm{~V}$. Tyurenkova, "Two regimes of a single n-heptane droplet combustion," Acta Astronaut. 163, 25-32 (2019).

${ }^{31}$ V. V. Tyurenkov, N. N. Smirnov, and V. M. Guendugov, "Analytical solution for a single droplet diffusion combustion problem accounting for several chain reaction stages," Acta Astronaut. 83, 208-215 (2013).

${ }^{32}$ F. E. Fendell, M. L. Sprankle, and D. S. Dodson, "Thin-flame theory for a fuel droplet in slow viscous flow," J. Fluid Mech. 26, 267-280 (1966).

${ }^{33}$ R. Kurose, H. Makino, S. Komori, M. Nakamura, F. Akamatsu, and M. Katsuki, "Effects of outflow from the surface of a sphere on drag, shear lift, and scalar diffusion," Phys. Fluids 15, 2338-2351 (2003).

${ }^{34}$ S. S. Sadhal and P. S. Ayyaswamy, "Flow past a liquid drop with a large nonuniform radial velocity," J. Fluid Mech. 133, 65-81 (1983).

${ }^{35}$ G. Gogos, S. S. Sadhal, P. S. Ayyaswamy, and T. Sundararajan, "Thin-flame theory for the combustion of a moving liquid drop: Effects due to variable density," J. Fluid Mech. 171, 121-144 (1986).

${ }^{36}$ M. A. Jog, P. S. Ayyaswamy, and I. M. Cohen, "Evaporation and combustion of a slowly moving liquid fuel droplet: Higher order theory," J. Fluid Mech. 307, 135-165 (1996).

${ }^{37}$ P. Balakrishnan, T. Sundararajan, and R. Natarajan, "Combustion of a fuel droplet in a mixed convective environment," Combust. Sci. Technol. 163, 77-106 (2001).

${ }^{38}$ H. Hara and S. Kumagai, "The effect of initial diameter on free droplet combustion with spherical flame," Symp. Combust. 25, 423-430 (1994).

${ }^{39}$ K. Pandey, S. Basu, V. Gautham, A. Potnis, and K. Chattopadhyay, "Self-tuning and topological transitions in a free-falling nanofuel droplet flame," Combust. Flame 220, 144-156 (2020).

${ }^{40}$ S. M. Al-Noman, S. K. Choi, and S. H. Chung, "Autoignition characteristics of laminar lifted jet flames of pre-vaporized iso-octane in heated coflow air," Fuel 162, 171-178 (2015).

${ }^{41}$ Y. S. Ko and S. H. Chung, "Propagation of unsteady tribrachial flames in laminar non-premixed jets," Combust. Flame 118, 151-163 (1999).

${ }^{42} \mathrm{~K}$. Bergeles, Y. Hardalupas, and A. M. K. P. Taylor, "On the transient flow inside and around a deforming millimetre class oil droplet falling under the action of gravity in stagnant air," Phys. Fluids 30, 013305 (2018).

${ }^{43} \mathrm{~K}$. Kumar and C. J. Sung, "Laminar flame speeds and extinction limits of preheated n-decane $/ \mathrm{O}_{2} / \mathrm{N}_{2}$ and n-dodecane $/ \mathrm{O}_{2} / \mathrm{N}_{2}$ mixtures," Combust. Flame 151, 209-224 (2007).

${ }^{44}$ A. Roshko, "On the drag and shedding frequency of two-dimensional bluff bodies,” Technical Note 3169 (1954). 